\title{
Clarifying the structure of low-lying states in ${ }^{72} \mathrm{Br}$
}

\author{
J. A. Briz $\odot,{ }^{1}$ M. J. G. Borge $\odot,{ }^{1}$ B. Rubio $\odot,{ }^{2}$ J. Agramunt $\odot,{ }^{2}$ A. Algora $\odot,{ }^{2,3}$ A. Y. Deo $\odot,{ }^{4,5}$ M. E. Estévez Aguado, ${ }^{2}$ \\ G. Farrelly $\odot,{ }^{4}$ L. M. Fraile $\odot,{ }^{6}$ W. Gelletly, ${ }^{4}$ A. Maira, ${ }^{1}$ E. Nácher $\odot,{ }^{2}$ A. Perea $\odot,{ }^{1}$ \\ Zs. Podolyák $\odot,{ }^{4}$ A. Poves $\odot,{ }^{7}$ P. Sarriguren $\odot,{ }^{1}$ and O. Tengblad ${ }^{1}$ \\ ${ }^{1}$ Instituto de Estructura de la Materia, CSIC, Serrano 113-bis, E-28006 Madrid, Spain \\ ${ }^{2}$ Instituto de Fisica Corpuscular, CSIC-Universidad de Valencia, E-46071 Valencia, Spain \\ ${ }^{3}$ Institute of Nuclear Research (ATOMKI), P. O. Box 51, H-4001 Debrecen, Hungary \\ ${ }^{4}$ Department of Physics, University of Surrey, Guildford GU2 7XH, Surrey, United Kingdom \\ ${ }^{5}$ Department of Physics, Indian Institute of Technology, Roorkee 247667, India \\ ${ }^{6}$ Grupo de Física Nuclear and IPARCOS, Universidad Complutense de Madrid, CEI Moncloa, E-28040 Madrid, Spain \\ ${ }^{7}$ Departamento de Física Teórica e IFT-UAM/CSIC, Universidad Autónoma de Madrid, E-28049 Madrid, Spain
}

(Received 31 May 2021; revised 5 October 2021; accepted 24 December 2021; published 25 January 2022)

\begin{abstract}
The spins and parities of low-lying states in ${ }^{72} \mathrm{Br}$ populated in the $\beta$ decay of ${ }^{72} \mathrm{Kr}$ have been studied via conversion electron spectroscopy. The measurements were carried out at ISOLDE using a miniorange spectrometer with $\mathrm{Si}(\mathrm{Li})$ and HPGe detectors for electrons and $\gamma$ ray detection. Results of the conversion coefficients corresponding to transitions deexciting 12 levels in ${ }^{72} \mathrm{Br}$ are reported. The multipolarities of the transitions are deduced and the spins and parities of the levels involved are discussed. From the multipolarities of the most intense transitions to the ground state, the spin and parity of the ${ }^{72} \mathrm{Br}$ ground state have been definitely established as $1^{+}$. The spin of the $101.2-\mathrm{keV}$ isomeric state is determined to be $3^{-}$. The level scheme is compared with mean-field and shell-model calculations and oblate deformation for the ${ }^{72} \mathrm{Br}$ ground state is deduced. No $E 0$ transitions have been found in ${ }^{72} \mathrm{Br}$. E0 transitions in the neighboring isobaric nuclei, ${ }^{72} \mathrm{Se}$ and ${ }^{72} \mathrm{Ge}$, have also been studied.
\end{abstract}

DOI: 10.1103/PhysRevC.105.014323

\section{INTRODUCTION}

The structure and decay of ${ }_{36}^{72} \mathrm{Kr}_{36}$ are of interest for a number of reasons. First, the nuclei with $N \simeq Z$ in the $A \approx$ $70-80$ region of the nuclear chart are of special interest as far as nuclear deformation is concerned. Nuclei along the $N=Z$ line with masses between 40 and 100 are predicted to change shape from spherical to oblate and prolate and back to spherical. In particular, shape coexistence [1,2] and shape mixing phenomena occur in this region. Second, the study of $N=Z$ nuclei can provide important information on $n p$ pairing as both neutrons and protons occupy similar orbitals and effects caused by $n p$ pairing are expected to show up in these systems [3-5]. Third, from the nuclear astrophysics point of view, ${ }^{72} \mathrm{Kr}$ is a waiting point in the rp process of stellar nucleosynthesis and plays a significant role in the understanding of $\mathrm{x}$-ray bursts emission curves [6-8].

The $\mathrm{Br}$ isotopes lie in the transition region between the weakly deformed As and Se isotopes and highly deformed $\mathrm{Kr}$ and $\mathrm{Rb}$. This motivates their study and, at the same time,

Published by the American Physical Society under the terms of the Creative Commons Attribution 4.0 International license. Further distribution of this work must maintain attribution to the author(s) and the published article's title, journal citation, and DOI. complicates the determination of their properties and the understanding of their structure, especially for the odd-odd isotopes such as ${ }^{72} \mathrm{Br}$.

Prior to the present work our knowledge of the level scheme of ${ }_{35}^{72} \mathrm{Br}_{37}$ was based on studies of the $\beta^{+} / \mathrm{EC}$ decay of ${ }^{72} \mathrm{Kr}$ [9-11] and fusion-evaporation reactions [12-16]. Two bands with opposite parity were identified in Ref. [12] with spins up to $I=9$. The knowledge of these bands was extended by Ulbig et al. [13] up to $I=15$ and included the determination of half-lives for many of these excited states. The work of Fotiades et al. [14] provided extra information on the previously determined positive-parity band extending the $\alpha=0$ partner up to $I=18^{+}$and the $\alpha=+1$ up to $I=21^{+}$. The organization of the positive-parity band in this way shows the expected staggering between signature partners of the bands similar to that found in the neighboring isotopes ${ }^{74,76,78} \mathrm{Br}$. No signature inversion at low spin was observed as expected from theoretical models for nuclei with less than 39 protons and neutrons [17].

Plettner et al. [15] using the ${ }^{40} \mathrm{Ca}\left({ }^{40} \mathrm{Ca}, \alpha 3 p 1 n\right){ }^{72} \mathrm{Br}$ reaction and the EUROBALL array for $\gamma-\gamma$ coincidences expanded the negative-parity band up to $I=\left(26^{-}\right)$and observed the signature inversion at $I \approx 16$. This observation for negative-parity bands is unique among odd-odd bromine isotopes. The cranked Nilsson Strutinsky (CNS) approach $[18,19]$ was used to reproduce the observed level spacing. In this theoretical framework the near-oblate and near-prolate 
shapes compete at low and intermediate spins. Collective near-oblate configurations were found to be lowest in energy up to spins $I=10-12$.

The most recently published fusion-evaporation study by O'Leary et al. [16] performed using the ${ }^{40} \mathrm{Ca}\left({ }^{36} \mathrm{Ar}, 3 p\right.$ 1 n) ${ }^{72} \mathrm{Br}$ reaction and the GAMMASPHERE detectors has significantly modified and extended the previous level scheme. Bands of positive and negative parity were identified up to spins $I=29$ and compared with the predictions of CNS calculations. The band structure was reorganized to be more in agreement with CNS expectations and left more low-energy states outside the band structure. They emphasized that ${ }^{72} \mathrm{Br}$ is a very challenging case for nuclear structure models since the CNS model works well for nuclei in this mass region, i.e., the isotone ${ }^{73} \mathrm{Kr}$ and the isotope ${ }^{73} \mathrm{Br}$, while has only moderate success in the case of ${ }^{72} \mathrm{Br}$.

In spite of the very extensive studies of the ${ }^{72} \mathrm{Br}$ structure, spin and parity assignments are only tentative due to the uncertain assignments of the spin and parity of the low-energy levels including the ground state.

The most recent and, at the same time, the most complete $\beta^{+} /$EC-decay spectroscopy work performed by Piqueras et al. [11] provides quite extensive information on the level scheme of ${ }^{72} \mathrm{Br}$ up to $2 \mathrm{MeV}$ for low-spin states based on $\gamma$-ray and $\gamma-\gamma$ coincidence measurements. Tentative spin-parities were assigned to some states in ${ }^{72} \mathrm{Br}$ based on $\beta$ feeding and $\log f t$ values.

The only experimental knowledge of transition multipolarities from conversion coefficients was obtained for the $101.2-\mathrm{keV}$ transition connecting the isomeric state $\left[T_{1 / 2}=\right.$ $10.6(3) \mathrm{s}]$ with the ${ }^{72} \mathrm{Br}$ ground state $\left[T_{1 / 2}=78.6(24) \mathrm{s}\right]$ and determined to be $M 2$, see Refs [12,20]. All of these results are included in the most recent nuclear data evaluation by Abriola et al. [21]. One should notice that this $M 2$ transition is one of the most hindered in the chart of nuclides with a hindrance factor with respect to the single-particle estimates larger than 60000 .

The spin-parity of the ${ }^{72} \mathrm{Br}$ ground state is originally reported from $\beta$-decay studies to be either $J^{\pi}=3^{+}$[22] or $1^{+}[9,11]$. The $3^{+}$assignment is based on the study of the ${ }^{72} \mathrm{Br} \rightarrow{ }^{72} \mathrm{Se}$ decay. The authors of Ref. [22] reported strong feeding from the ${ }^{72} \mathrm{Br}$ ground state to the two lowest $2^{+}$states $[20(2) \%$ to the $1316.7-\mathrm{keV}$ and $23.2(28) \%$ to the $862.0-\mathrm{keV}$ levels] and significant feeding to the lowest $4^{+}$state $[5.0(9) \%$ to the $1639.6-\mathrm{keV}$ level] in ${ }^{72} \mathrm{Se}$. On the contrary, the $1^{+}$ assignment $[9,11]$ is based on relative intensities of $\gamma$-ray transitions in the decay of ${ }^{72} \mathrm{Kr} \rightarrow{ }^{72} \mathrm{Br}$ in comparison with those belonging to the daughter decay ${ }^{72} \mathrm{Br} \rightarrow{ }^{72} \mathrm{Se}$. The $J^{\pi}=\left(3^{+}\right)$assignment was retained in all fusion-evaporation studies.

Due to the discrepancies encountered in the determination of spin-parity values of the low-energy excited states in ${ }^{72} \mathrm{Br}$ we have decided to determine the conversion electrons of the transitions from states populated in the $\beta^{+} / \mathrm{EC}$ decay of ${ }^{72} \mathrm{Kr}$ with the aim of obtaining information on the low-energy excitation structure of ${ }^{72} \mathrm{Br}$. This is timely as the controversial ${ }^{72} \mathrm{Kr}$ ground-state deformation has been determined to be oblate from different probes: electric-monopole decay of the $0^{+}$isomer in ${ }^{72} \mathrm{Kr}$ [23], total absorption spectroscopy based on the $\mathrm{B}(\mathrm{GT})$ distribution in ${ }^{72} \mathrm{Kr} \beta^{+} / \mathrm{EC}$ decay [24], and $B(E 2)$ values [25,26].

The paper is organized as follows. Section II describes the experimental setup with emphasis on the calibrations as we compare $\gamma$-ray and electron singles spectra with different miniorange configurations. In Sec. III, the analysis process is presented while in Sec. IV the results for the conversion coefficients and the resulting spin-parity assignments are discussed. Section V presents a comparison of our results with theoretical calculations and Sec. VI presents the results obtained in the search for $E 0$ transitions. To finish, Sec. VII summarizes the paper and presents the main conclusions.

\section{EXPERIMENTAL SETUP}

The experiment was performed at the ISOLDE facility at CERN, in Geneva (Switzerland). A $1.4-\mathrm{GeV}$ proton beam delivered from the CERN PS-Booster impinged on a $26-\mathrm{g} / \mathrm{cm}^{2}$ $\mathrm{Nb}$ target connected to a plasma ion source by a cold line to remove nonvolatile species. The reaction products were accelerated by a $60-\mathrm{kV}$ voltage toward the extraction electrode. The ${ }^{72} \mathrm{Kr}$ beam was separated in the high resolution mass separator and then conducted to the measurement station. There the ${ }^{72} \mathrm{Kr}$ beam was collected and transported by means of a magnetic tape to the measurement point in periodic cycles of $33.6 \mathrm{~s}$ (1 supercycle of 28 pulses spaced $1.2 \mathrm{~s}$ each other). During the measurement, 14 of the total 28 proton pulses which form the CERN PS-Booster supercycle were delivered to the ISOLDE Nb target.

Surrounding the sample a high-purity germanium detector (HPGe) and an electron spectrometer were placed to determine the intensity of $\gamma$ rays and conversion electrons, respectively. The average ${ }^{72} \mathrm{Kr}$ yield was estimated for measurements with a cycle of $33.6 \mathrm{~s}$ using the most intense $\gamma$-ray transitions in the decay of ${ }^{72} \mathrm{Kr}$ and the known branching ratios, i.e., the $124.4-, 162.8-, 310.3-\mathrm{keV}$, the doublets $(414.7+415.5)-\mathrm{keV}$, and $(576.2+577.2)-\mathrm{keV}$ lines. We determine a yield of $5400{ }^{72} \mathrm{Kr}$ ions/s in the experimental setup which translates into an approximate yield of 9000 ions $/ \mu \mathrm{C}$.

The observed isobaric chain from the decay of ${ }^{72} \mathrm{Kr}$ stops at the decay of ${ }^{72} \mathrm{Se}\left(T_{1 / 2}=8.4\right.$ days $)$ as we are using a tape to remove the activity. However, from the observation of relative intensities of transitions in ${ }^{72} \mathrm{Ge}$ we have identified the presence of ${ }^{72}$ As. We estimate the incoming ${ }^{72}$ As contaminant to be roughly $3 \%$ of the ${ }^{72} \mathrm{Kr}$ beam.

\section{A. The miniorange spectrometer}

The electron spectrometer consists of miniorange magnets and a $300 \mathrm{~mm}^{2}$, 4-mm-thick $\mathrm{Si}(\mathrm{Li})$ detector cooled down to liquid nitrogen temperature. It follows the same principle described in Ref. [27]. The miniorange magnet, as shown in Fig. 1, has a central tungsten piece in order to avoid direct $\gamma$ and $x$ rays from the source reaching the $\mathrm{Si}(\mathrm{Li})$ detector. There are several sets of magnets in order to optimize the transmission of the detector for different electron energy ranges. Each configuration is identified using the label NT/d/D where $\mathrm{N}$ is the number of magnets, $\mathrm{T}$ is the type of magnet, which could be of two types $\mathrm{A}$ and $\mathrm{B}$ with the main difference being the 


\section{B/d/D mini-orange configuration}

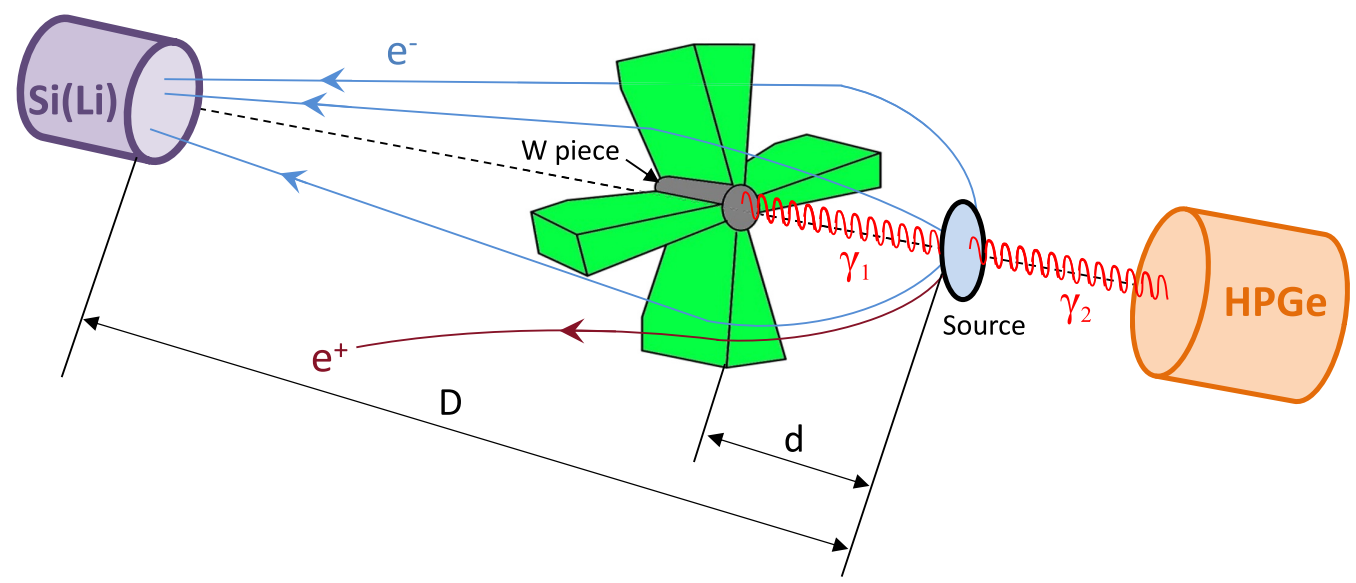

FIG. 1. Schematic view of the experimental setup including the miniorange spectrometer. The central tungsten piece prevents direct $\gamma$ and $\mathrm{x}$ rays from the source reaching the $\mathrm{Si}(\mathrm{Li})$ detector. The magnets are used to focus (defocus) electrons (positrons) toward the $\mathrm{Si}(\mathrm{Li})$ detector. Different sets of magnets and distances were used to optimize electron transmission in different energy ranges, as shown in Fig. 2. A HPGe detector is installed at $180^{\circ}$ to the miniorange for the detection of $\gamma$ rays.

size and, therefore, the magnetic field created, $\mathrm{d}$ (in $\mathrm{mm}$ ) is the source to miniorange distance and $\mathrm{D}$ is the source-Si(Li) detector distance (in $\mathrm{mm}$ ). Distances $\mathrm{d}$ and $\mathrm{D}$ are indicated in Fig 1.

The $\mathrm{Si}(\mathrm{Li})$ detector includes a $260 \mu \mathrm{g} / \mathrm{cm}^{2}$ polyethylene window, aluminized on both faces and placed $4 \mathrm{~mm}$ in front of the detector. The $\mathrm{Si}(\mathrm{Li})$ detector is cooled-down to liquid nitrogen temperature and showed an energy resolution of about $2.0 \mathrm{keV}$ for electrons in the energy range from 25 to $1000 \mathrm{keV}$. For a more complete description of the spectrometer see [28].

The efficiency curve for each miniorange configuration, also known as the transmission curve, was determined using transitions with well-known conversion coefficients either from an external ${ }^{207} \mathrm{Bi}$ source or by internally produced ${ }^{74,75,76} \mathrm{Kr}$ sources.

The electron efficiency curves for the different settings were obtained using Eq. (1),

$$
\tau_{e}\left(E_{\gamma}\right)=\frac{I_{e}(\text { detected })}{I_{e}(\text { emitted })}=\frac{A_{e} / t_{e}}{\alpha \times A_{\gamma} /\left[\epsilon\left(E_{\gamma}\right) \times t_{\gamma}\right]},
$$

where the measured electron and $\gamma$-ray peak areas, $A_{e}$ and $A_{\gamma}$, are divided by the respective live times of each detection system, $t_{e}$ and $t_{\gamma}$, the HPGe detector efficiency, $\epsilon\left(E_{\gamma}\right)$ and the $\alpha$ conversion coefficients are taken from the literature. The resulting transmission curves are shown in Fig. 2. For the $4 \mathrm{~B} / 8 / 85$ miniorange configuration the values of conversion coefficients for transitions in ${ }^{75} \mathrm{Br}$ and ${ }^{75} \mathrm{Se}$ were taken from Refs. [29-31]. For the 6A/8/60 and 6A/8/45 miniorange configurations, conversion coefficients for transitions in ${ }^{207} \mathrm{~Pb}[32]$ and ${ }^{72,74} \mathrm{Se}[33,34]$ were used. For the $3 \mathrm{~B} / 8 / 45$ configuration, the transmission curve uses conversion coefficients for transitions in ${ }^{76} \mathrm{Br}$ [35], shown in Fig. 2 in red, and some transmission values taken from Ref. [29], in blue. As shown in Fig. 2 the combined data has allowed us to obtain a reliable transmission curve. A $20 \%$ uncertainty has been assigned for all the transmission values.

\section{B. Gamma-ray detector}

A HPGe detector was used in the measurements to detect $\gamma$ and $\mathrm{x}$ rays. The HPGe detector had $38 \mathrm{~cm}^{2}$ frontal area and a thickness of $2.55 \mathrm{~cm}$ and was located $2 \mathrm{~cm}$ away from the measurement point, covering a solid angle of $25 \%$ of $4 \pi$. The 0.3-mm-thick Be window of the detector allowed the detection of the $\mathrm{Br} \mathrm{x}$ rays down to $13 \mathrm{keV}$, see Fig. 4. It exhibited an energy resolution of $0.85 \mathrm{keV}$ at $121-\mathrm{keV}$ energy and an effective energy range of 10-1250 keV.

A good absolute photopeak efficiency calibration is required in order to extract reliable absolute intensities for every transition. To this end, a careful efficiency calibration, including a GEANT4 simulation, was performed for the HPGe detector. The resulting efficiency curve is shown in Fig. 3. Standard calibration sources of ${ }^{241} \mathrm{Am},{ }^{133} \mathrm{Ba}$, and ${ }^{152} \mathrm{Eu}$ were used for calibration. The experimental data were fitted using two efficiency functions. One is optimised for low energies and is shown in Eq. (2) [37]. The other is valid for higher energies and is given in Eq. (3) [38]. The value of the energy at which both functions are connected was determined to be 146 $\mathrm{keV}$ by imposing the condition of continuity and smoothness of the resulting function.

$$
\begin{aligned}
\varepsilon(E) & =b_{1} \cdot \exp \left(b_{2} \cdot E^{b_{3}}\right)\left[1-\exp \left(b_{4} \cdot E^{b_{5}}\right)\right] \\
\ln \varepsilon(E) & =2 \cdot\left(a_{1}+a_{2} x+a_{3} x^{2}\right) \cdot \frac{\operatorname{atan}\left[e^{\left(a_{4}+a_{5} x+a_{6} x^{3}\right)}\right]}{\pi}-25,
\end{aligned}
$$

where $x=\ln (E)$ and $a_{i}$ and $b_{i}$ are constants to be obtained from the fit to the experimental data. The transition energy $E$ is expressed in $\mathrm{keV}$ in both expressions. A $10 \%$ uncertainty is considered for all the efficiency values of the HPGe detector.

\section{ANALYSIS}

The conversion coefficients $\left(\alpha=I_{e} / I_{\gamma}\right)$ were determined from the singles spectra taken with the $\mathrm{Si}(\mathrm{Li})$ detector for 

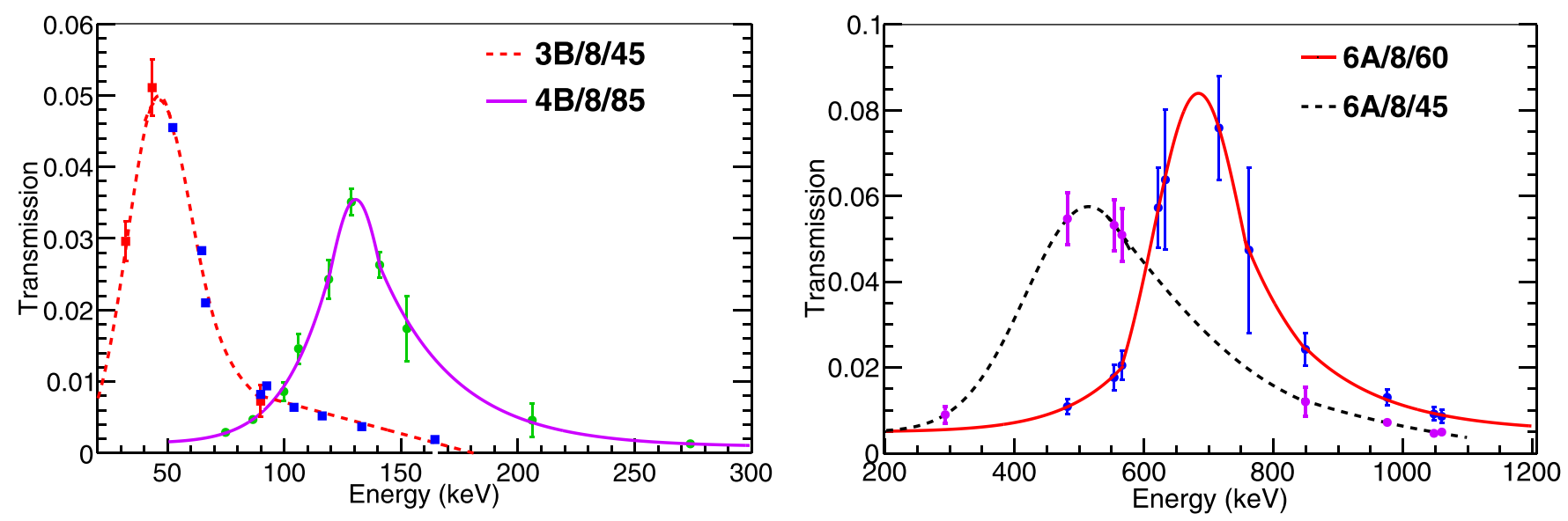

FIG. 2. Electron efficiency curves for the various configurations of magnets and distances used in the experiment in order to maximize the transmission for different energy ranges.

conversion electrons and the HPGe detector for $\gamma$ rays. Figure 4 shows part of the conversion electron and $\gamma$-ray spectra for the $4 \mathrm{~B} / 8 / 85$ configuration of the miniorange spectrometer as an example. The identification of electron transitions in the $\mathrm{Si}(\mathrm{Li})$ detector was made by taking into account the binding energies of electrons from the different atomic shells in Bromine, i.e., being $13.5 \mathrm{keV}$ for the $\mathrm{K}$ shell, being $1.8 \mathrm{keV}$ for the $\mathrm{L} 1$ subshell and $1.6 \mathrm{keV}$ for the $\mathrm{L} 2$ and L3 subshells, and $0.3 \mathrm{keV}$ and lower for the $\mathrm{M}$ shell. Therefore, the energy differences between the L- and M-shell lines are about $1.5 \mathrm{keV}$, which is smaller than the energy resolution of the $\mathrm{Si}(\mathrm{Li})$ detector. As a consequence, these components could not be resolved and we obtained a coefficient for those electron shells together ( $\mathrm{L}$ and $\mathrm{M}$ ), which we call the "LM shell" coefficient.

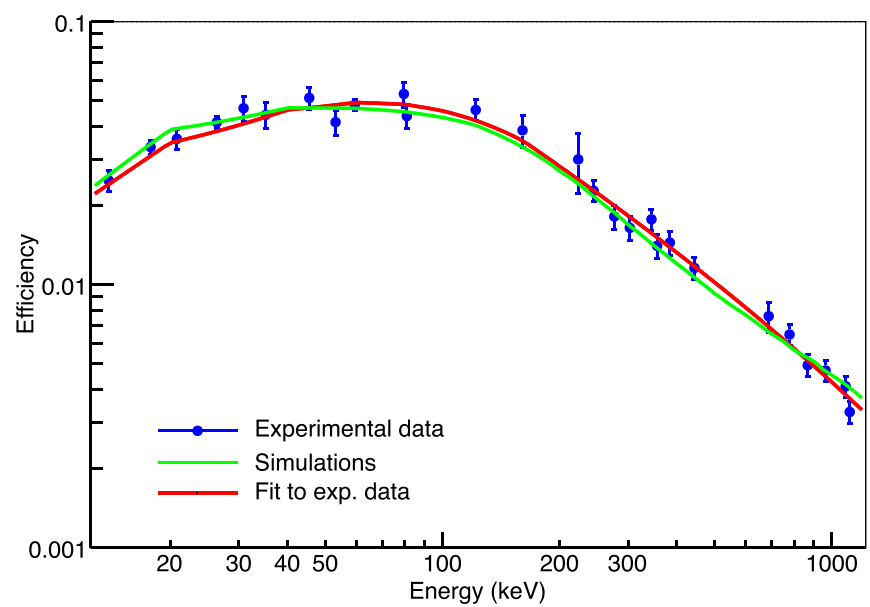

FIG. 3. The measured efficiency calibration curve for the HPGe detector. The experimental data are represented with blue dots. The plot shows two different curves: The red line is the fit to the combination of Eqs. (2) and (3) to the experimental points and the green line is the simulated efficiency of the detector carried out with GEANT4 [36].
The expression used to obtain the conversion coefficients is given in Eq. (4),

$$
\alpha=\frac{I_{e}}{I_{\gamma}}=\frac{A_{e} /\left(\tau_{e} \cdot t_{e}\right)}{A_{\gamma} /\left(\epsilon_{\gamma} \cdot t_{\gamma}\right)},
$$

where $A_{e}$ and $A_{\gamma}$ are the electron and $\gamma$-ray peak areas, $t_{e}$ and $t_{\gamma}$ are the live times for the electron and $\gamma$-ray detection systems, and $\tau_{e}$ and $\varepsilon_{\gamma}$ the electron and $\gamma$-ray detector efficiencies at the energies under consideration. The areas of the $\gamma$-ray peaks were obtained by fitting to a Gaussian plus linear background function. The electron peaks have a tail at lower energies due to the losses in the aluminized-mylar window of the $\mathrm{Si}(\mathrm{Li})$ detector and in the transport tape in order to exit from the depth reached by the implanted ${ }^{72} \mathrm{Kr}$ ions. To determine more accurately the shape of the electron peak and with that its area we use a compound function taken from Ref. [39] and given in Eq. (5),

$$
\begin{aligned}
F(x)= & c_{1} \frac{1}{\sigma \sqrt{2 \pi}} e^{-(x-\mu)^{2} / 2 \sigma^{2}} \\
& +c_{2} e^{\left[(x-\mu) / \beta+\sigma^{2} /\left(2 \beta^{2}\right)\right]} \operatorname{erfc}\left(\frac{x-\mu}{\sqrt{2} \sigma}+\frac{\sigma}{\sqrt{2} \beta}\right) \\
& +c_{3} \operatorname{erfc}\left(\frac{x-\mu}{\sqrt{2} \sigma}\right) .
\end{aligned}
$$

Equation (5) includes a Gaussian term, a skewed Gaussian term to take into account the energy losses of electrons, and a third term modeling the background.

For a more detailed description of the methods used to calibrate the detectors see Ref. [40].

\section{RESULTS}

The experimental $\gamma$-ray spectra were analyzed to determine the relative transition intensities listed in Table I. The adopted values of the intensities obtained in this work were derived from the sum of the $\gamma$-ray spectra obtained from the four measurements made with different miniorange configurations. The $\gamma$-ray transition intensities are compared 

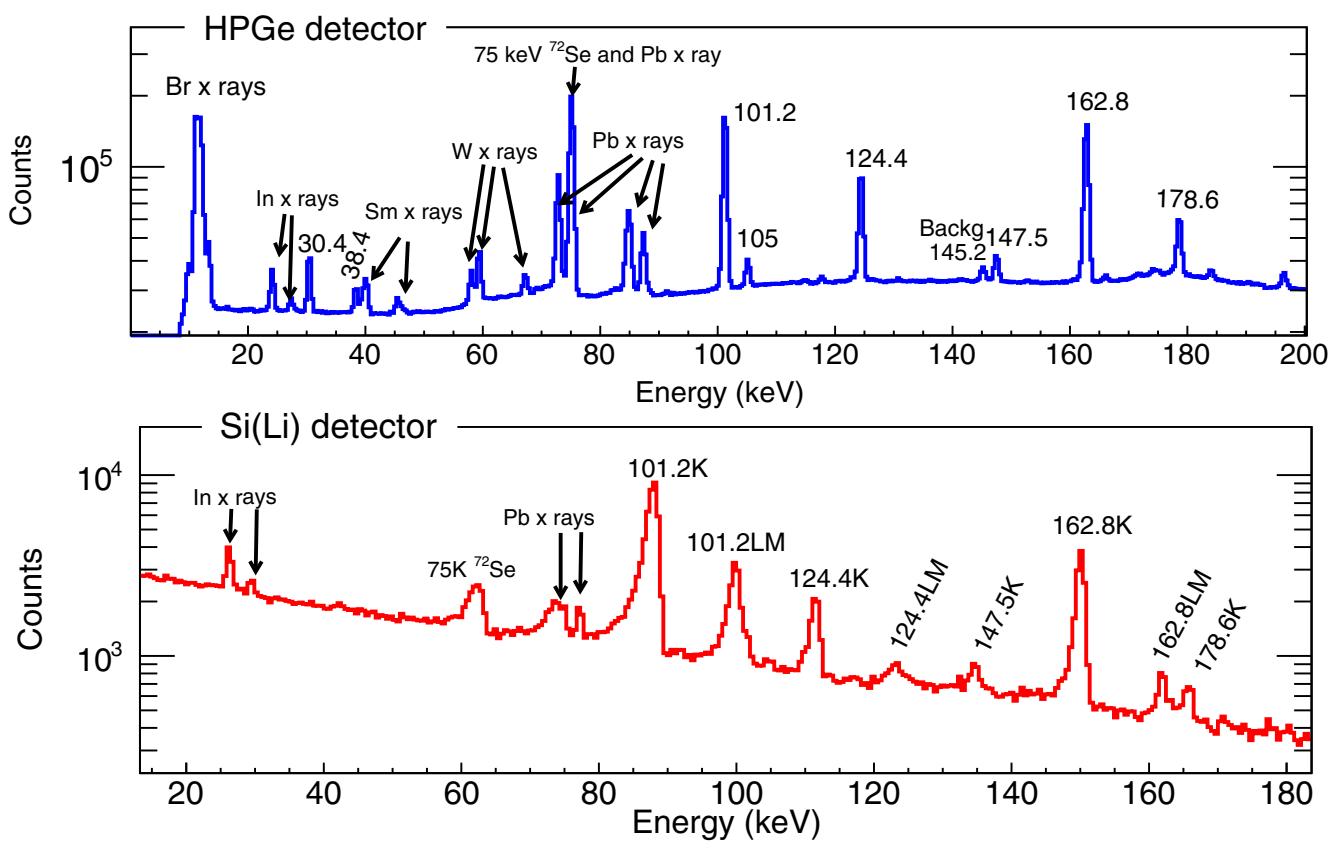

FIG. 4. Spectra taken with HPGe (top) and $\mathrm{Si}(\mathrm{Li}$ ) (bottom) detectors with the configuration 4B/8/85. The x rays from indium (In), lead $(\mathrm{Pb})$, tungsten $(\mathrm{W})$, and samarium $(\mathrm{Sm})$ are seen. The tungsten is present in the central piece and samarium in the blades of the miniorange magnet. Their $\mathrm{x}$ rays are only seen in the HPGe detector as it is facing the activated side of the magnet. The indium and lead $\mathrm{x}$ rays come from the activation of the lateral shielding pieces in the experimental chamber. They are seen in both detectors since they are not stopped by the tungsten piece of the miniorange. An additional 145.2-keV line is present in the background as well but its origin is not yet identified. Note that the binding energies for K-shell, L-shell, and M-shell electrons in bromine are $13.5,1.6-1.8$, and $\leqslant 0.3 \mathrm{keV}$, respectively.

TABLE I. List of the most intense $\gamma$ rays measured in our study in comparison with those of Ref. [21]. The 310.3-keV line is used for normalization. Intensities are given per 100 decays. Adopted values from this work are obtained from the summed $\gamma$ spectrum of the four independent measurements done with different miniorange configurations. The errors are based on statistical uncertainties and fitting approximations. *Obtained assuming a conversion coefficient of $\alpha=20.7$. ${ }^{\dagger}$ This intensity is apparent. NS indicates that the transition has not been seen. ${ }^{\ddagger} \gamma$-line contaminated by the $252.0-\mathrm{keV} E 1$ transition in ${ }^{76} \mathrm{Br}$. ${ }^{\S}$ Determined from the spectrum obtained by summing three of the four measurements.

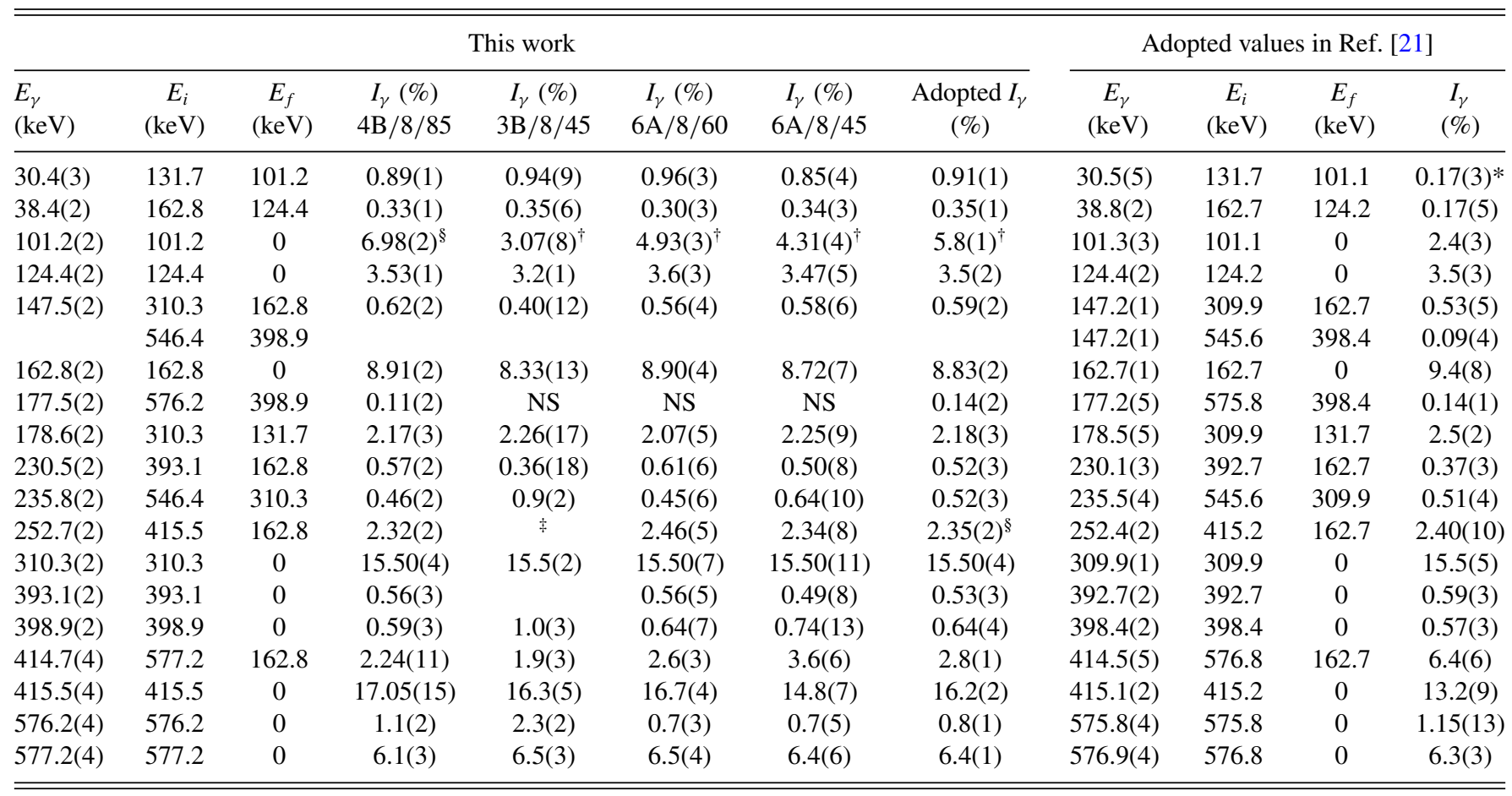


TABLE II. Conversion coefficients for the $\mathrm{K}$ and LM shells of transitions in ${ }^{72} \mathrm{Br}$. Experimental conversion coefficients are given in column 4. The theoretical predictions are listed in the following 6 columns. The dominant multipolarity for each transition is given in column 11. For the transitions with mixed multipolarity the mixing ratio $|\delta|$ is listed in column 12 . Previous results are given in the last column where the multipolarity assignments are deduced from intensity balance arguments in Ref. [11] unless indicated. D147.5 corresponds to the doublet with both $\gamma$ rays of 147.5(2) keV energy. D415 and D577 doublets are formed by the 414.7- and 415.5-keV transitions, and the 576.2- and 577.2-keV transitions, respectively.

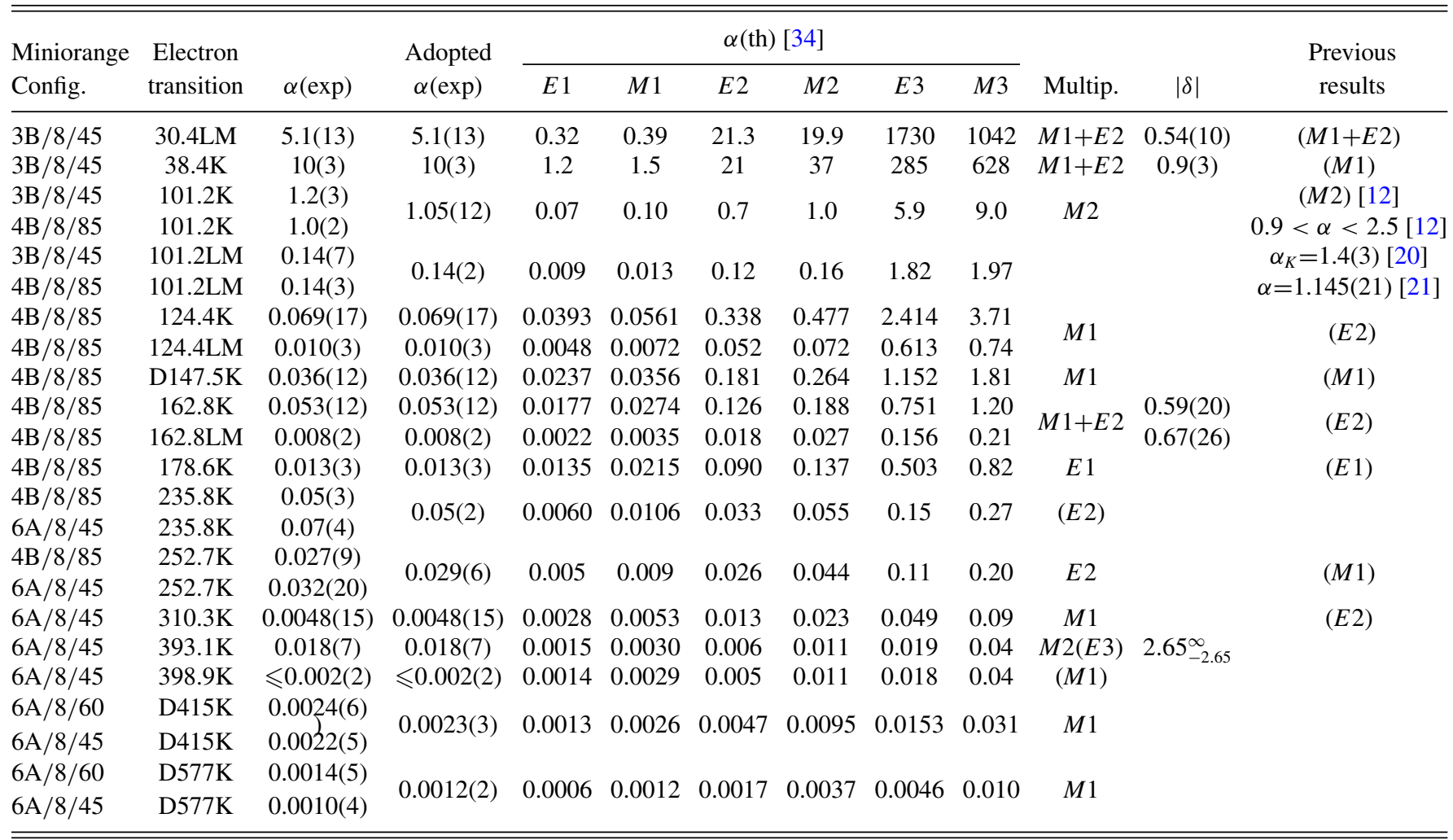

with the adopted values given in the last nuclear data evaluation [21].

In general terms, the intensities obtained in our study for all the measurements performed with different miniorange configurations agree among themselves and with the values from Ref. [21]. For the normalization of all the $\gamma$-ray intensities, the $310.3-\mathrm{keV}$ transition is taken as a reference.

The doublet at $147.5(2) \mathrm{keV}$ is not resolved and the two transitions are treated together. The 414.7- and 415.5-keV transitions are only partially resolved in the $\gamma$-ray spectra and their relative intensities were determined by a fit to two Gaussian functions. The same procedure was applied to the 576.2- and 577.2-keV transitions, which were also not fully resolved.

There is a significant discrepancy of a factor of 5 in the 30.4-keV $\gamma$-ray relative intensity between the value given in Ref. [21] and our work, see Table I. However, the value in Ref. [21] is obtained assuming an $\alpha_{\text {Tot }}=20.7$ derived in Ref. [11] from intensity balance considerations. Using the conversion coefficient found in this work, see Table II, this discrepancy reduces to a factor 1.5.

\section{A. Conversion coefficients}

The experimental values for the conversion coefficients are shown in Table II. The experimental conversion coefficients are based on the singles $\gamma$-ray and electrons spectra. When two transitions could not be resolved, a summed conversion coefficient was determined. They are labeled in column 2 of Table II by placing a D before the transition energy and atomic shell involved. In cases where the same transition was obtained from measurements with different miniorange configurations, for example for the cases of $101.2 \mathrm{~K}$ or D415K, the adopted values for the conversion coefficients are obtained from the weighted average of the values measured for each configuration. The uncertainties for each coefficient have been determined by error propagation of the variables involved in Eq. (4).

The conversion coefficient values are compared with theoretical calculations based on the Dirac-Fock calculations using the "frozen orbital" approximation from Ref. [34] for the different multipolarities and they are illustrated in Figs. 5 and 6 . The results on conversion coefficients of low-energy transitions in ${ }^{72} \mathrm{Br}$ are summarized in Table II. They provide the basis for the discussion of the multipolarities of the transitions and our conclusions concerning the spins and parities of the levels in ${ }^{72} \mathrm{Br}$.

\section{B. Spin-parity assignments}

Many of the states discussed here have been observed in both $\beta^{+} / \mathrm{EC}$ decay and fusion-evaporation reactions. 

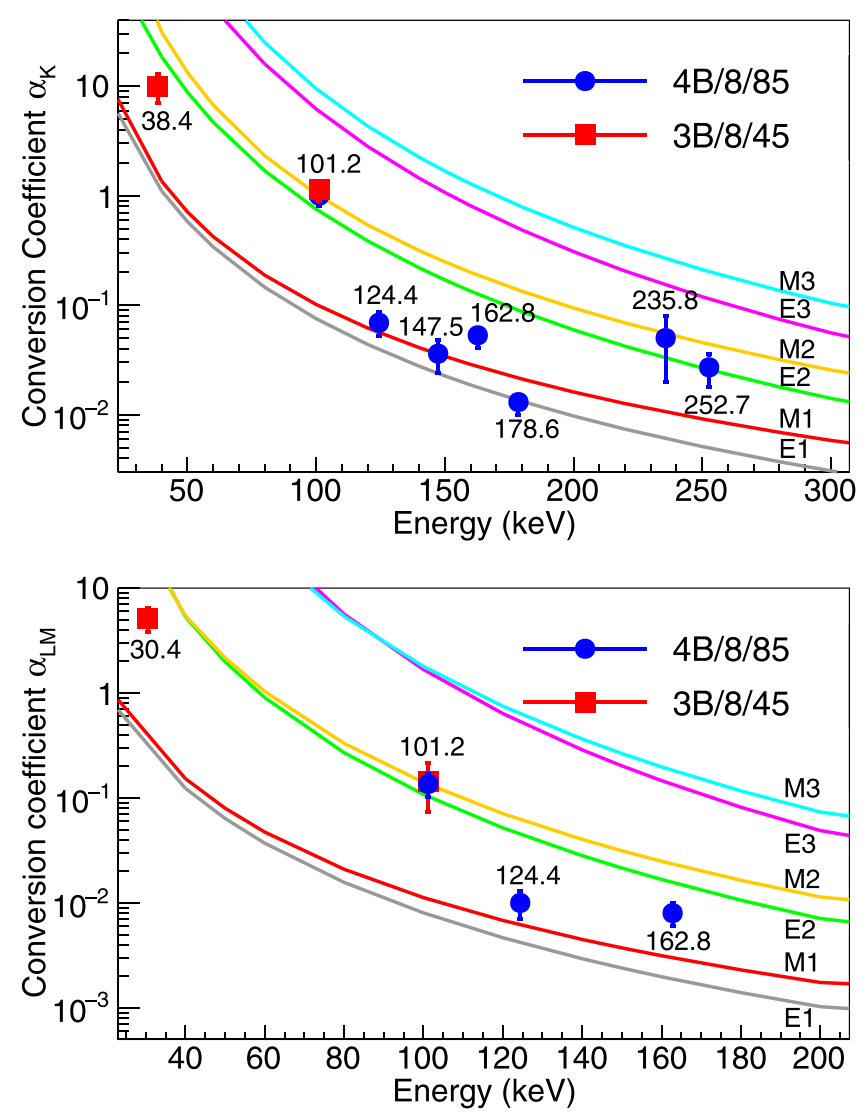

FIG. 5. Experimental conversion coefficients for K-shell (top) and LM-shell (bottom) transitions of low energy measured with the miniorange $4 \mathrm{~B} / 8 / 85$ and $3 \mathrm{~B} / 8 / 45$ configurations. The comparison with the theoretical calculations from Ref. [34] is presented for the different multipolarities. Labels indicate the transition energy in $\mathrm{keV}$.

Nevertheless, most of these states are not considered part of any rotational band and their spin-parities have not been previously established. In what follows the spin and parity

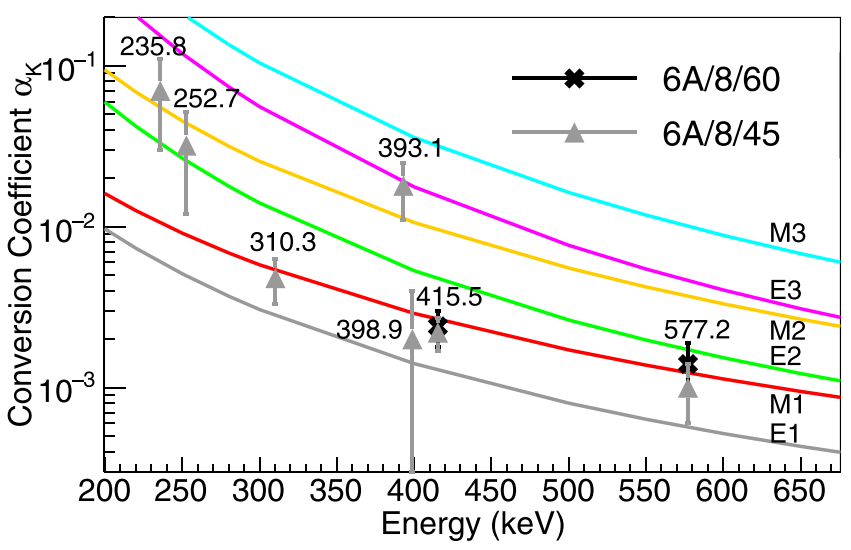

FIG. 6. Experimental conversion coefficients for K-shell transitions obtained in the high energy range with the miniorange $6 \mathrm{~A} / 8 / 60$ and $6 \mathrm{~A} / 8 / 45$ configurations. The comparison with the theoretical calculations from Ref. [34] is presented for the different multipolarities. Labels indicate the transition energy in $\mathrm{keV}$. assignments are based on the multipolarities determined from the conversion coefficients measured in this work.

\section{Ground state}

The spin of the ${ }^{72} \mathrm{Br}$ ground state was not firmly established in previous works. The assignment of $1^{+}$was suggested in Refs. [9-11] due to strong direct $\beta$ feeding deduced from the $\beta^{+} /$EC decay of ${ }^{72} \mathrm{Kr}$. Since the ${ }^{72} \mathrm{Kr}$ ground state is $0^{+}$, the strong direct feeding suggests the assignment of $1^{+}$for the ground state of ${ }^{72} \mathrm{Br}$ populated by an allowed Gamow-Teller transition.

In contrast, in Ref. [22] the ${ }^{72} \mathrm{Br}$ ground state was assigned $3^{+}$based on the direct $\beta$ feeding of two $2^{+}$and one $4^{+}$states in ${ }^{72} \mathrm{Se}$. However, the $\log f t$ of the transition to the $4^{+}$state at $1639.6 \mathrm{keV}$ was reported to be 7.01 , which lies at the upper limit for an allowed GT transition. This spin value was retained for all the fusion-evaporation studies.

Three levels at 310,415 , and $577 \mathrm{keV}$ were identified as the most intensely fed in the $\beta$ decay of ${ }^{72} \mathrm{Kr}$ [9-11]. The latest values for the feeding for these states are 16.42, 15.79, and $13.06 \%$ [11] corresponding to $\log f t$ values of $4.83,4.79$, and 4.78 , respectively. The systematics of $\log f t$ values given in Ref. [41] shows that forbidden transitions with log $f t$ below 5.0 have not yet been found. Thus, it was assumed that these three transitions following the decay of ${ }^{72} \mathrm{Kr}$ were allowed $0^{+} \rightarrow 1^{+}$transitions and these three levels had $1^{+}$spin-parity.

The conversion coefficients of the three transitions connecting these three $1^{+}$levels with the ${ }^{72} \mathrm{Br}$ ground state have been measured in this work indicating $M 1$ character for the three cases, see Table II and Fig. 6. Based on the multipolarities of these transitions starting at $1^{+}$levels, the only possible spins for the ground state are $0^{+}, 1^{+}$, or $2^{+}$. Therefore, the previously proposed assignment of $3^{+}$is excluded.

The $\beta$ decay $0^{+} \rightarrow 0^{+}$transition is forbidden for even-even $N=Z$ nuclei. However, previous $\beta$-decay studies indicated direct feeding to the ${ }^{72} \mathrm{Br}$ ground state at the level of $54 \%$ [9], 9\% [10], and 35(3)\% [11]. Therefore, the $0^{+}$possibility is discarded. Regarding the $2^{+}$option, the systematics on $\log f t$ values for second forbidden transitions $\Delta J=2$, $\Delta \pi=n o$ transitions gives a minimum value of $\log f t=10.6$ providing a maximum $\beta$ feeding of $4 \times 10^{-4}$. Thus $2^{+}$can be ruled out as well. Our results definitely support $1^{+}$as the ground-state spin-parity. This value was already adopted in the more recent nuclear data evaluation [21]. In addition, the $1^{+}$assignment, contrary to the previous $3^{+}$, is compatible with the low value of the ground-state magnetic moment measured to be $\mu=0.55(21) \mu_{N}$ in Ref. [20] and $\mu=0.60(10) \mu_{N}$ in Ref. [42].

The spin and parity of the ${ }^{72} \mathrm{Br}$ ground state has now been experimentally established in an independent way. This fixes the basis for the assignment of the spins and parities of the excited states including the rotational bands which had been built on top of the $\left(3^{+}\right)$assignment, see Refs. [12-16].

\section{101.2-keV isomeric state}

The retarded 101.2-keV transition was assumed to connect an isomeric state with the ground state [12]. The relative position of the ground and isomeric state has been recently 


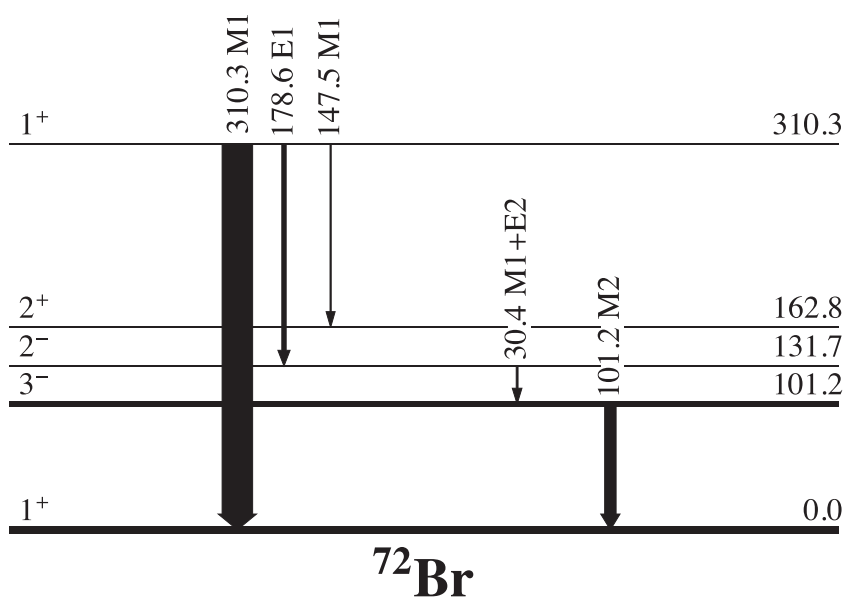

FIG. 7. Partial level scheme of ${ }^{72} \mathrm{Br}$ for the discussion of the spin and parity of the isomeric at $101.2(2) \mathrm{keV}\left[T_{1 / 2}=10.6(3) \mathrm{s}\right]$ and 131.7-keV states. The thickness of transition arrows follows the $\gamma$ transition intensities given in Table I.

measured using the Penning trap technique establishing an excitation energy of 101.3(15) keV [43] in agreement with our value deduced from the 101.2(2)-keV $\gamma$-ray energy.

The 101.2-keV isomeric $\left[T_{1 / 2}=10.6(3) \mathrm{s}\right]$ state deexcites by a single $\gamma$-ray transition to the ground state as reported in Refs. [11-13,20]. The spin-parity of this state was proposed to be $\left(1^{-}\right)$in Ref. [12]. This value was deduced from an estimate of the conversion coefficient, which was $0.9<\alpha<2.5$ as obtained from intensity balance, corresponding to an $M 2$ multipolarity, and the assumed $3^{+}$spin-parity of the ground state [22]. A measurement of the conversion coefficient was mentioned by Griffiths et al. in Ref. [20] with a value of $\alpha_{K}(\exp )=1.4(3)$ corroborating the previous $M 2$ multipolarity assignment.

The 101.2-keV state was assumed to be the bandhead of a negative-parity band $[12,13]$. However, the 101.2- and 131.7$\mathrm{keV}$ states were discarded as members of the negative-parity band in recent fusion-evaporation studies $[15,16]$.

The conversion coefficients obtained in this work for the 101.2-keV transition were measured using two miniorange configurations, $3 \mathrm{~B} / 8 / 45$ and $4 \mathrm{~B} / 8 / 85$. The results can be seen in Table II and graphically in Fig. 5. The adopted values are $\alpha_{K}(\exp )=1.05(12)$ and $\alpha_{\mathrm{LM}}(\exp )=0.14(2)$ in agreement with the theoretical values for an $M 2$ transition of 0.987 and 0.1578 , respectively. Therefore, we confirm the previous assignment of $M 2$ multipolarity to the $101.2-\mathrm{keV}$ transition and provide a precise experimental determination of the conversion coefficient.

Considering the $M 2$ multipolarity of the transition and the established $1^{+}$character for the ground state, we assign a $3^{-}$ spin-parity for the isomeric $101.2-\mathrm{keV}$ state. Figure 7 shows a partial level scheme including the 101.2-keV state.

From the Weisskopf estimates, the half-life of a 101.2-keV transition of $M 2$ multipolarity should be $T_{1 / 2}$ (W.u.) $=3.1 \times$ $10^{7} \times E_{\gamma}^{-5} \times A^{-2 / 3}=1.7 \times 10^{-4} \mathrm{~s}$ [44]. Although it fulfills the condition for $M 2$ transitions of $T_{1 / 2}(\exp ) / T_{1 / 2}$ (W.u.) $\geqslant 1$ [45] this transition presents a hindrance factor larger than

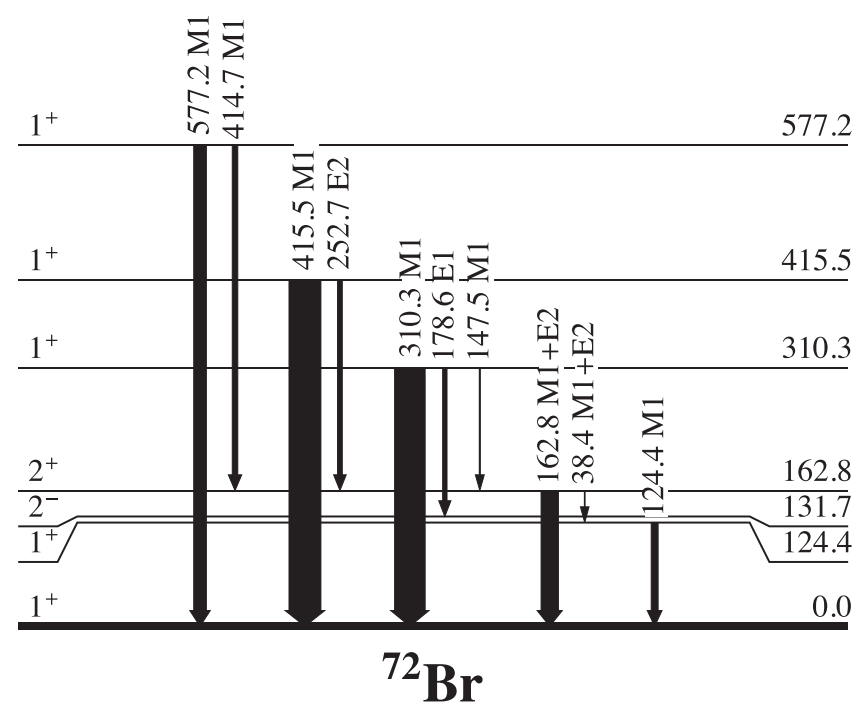

FIG. 8. Levels and transitions involved in the discussion of the spin-parity assignment of the 124.4-keV, 162.8-keV, 310-keV, $415.5-\mathrm{keV}$, and $577.2-\mathrm{keV}$ states in ${ }^{72} \mathrm{Br}$. The thickness of transition arrows follows the $\gamma$ transition intensities from this work given in Table I.

60000 that is surprisingly high. In this region of the chart of nuclides, the sudden changes of shapes facilitate the presence of highly hindered $M 2$ transitions. The more outstanding case in this region is ${ }^{70} \mathrm{Cu}$ with a $1^{+}$isomeric state at $242.2 \mathrm{keV}$ with a half-life of $6.6 \mathrm{~s}$ with a $\beta$-decay branch of $93.2 \%$ and deexciting via a retarded $M 2$ transition to a $3^{-}$state at 101.3$\mathrm{keV}$ with a probability of $6.8 \%$. The hindrance factor for this transition is even larger than in our case, about 200000. Considering the large hindrance factor for the $101.2 \mathrm{-keV} \mathrm{M2}$ transition, similar to that in the ${ }^{70} \mathrm{Cu}$ case, a $\beta$-decay branch for the isomeric state cannot be discounted. Assuming that the $3^{-}$isomeric state has a non-negligible $\beta$-decay branch, a $\beta$ feeding of the order of $5 \%$ to the $4^{+} 1639.6-\mathrm{keV}$ state in ${ }^{72} \mathrm{Se}$, as reported in Refs. [22,46], is plausible.

Another case is ${ }^{76} \mathrm{Br}$, where an $M 2$ transition of $57.1 \mathrm{keV}$ connects a (4) ${ }^{+}$state at $102.6 \mathrm{keV}$ with a (2) ${ }^{-}$state at 45.5 $\mathrm{keV}$ with a half-life of $1.31 \mathrm{~s}$. The hindrance factor for this case is only about 500 .

A survey of M2 transitions was done in Ref. [47] in 1967 for low-lying nuclear states. The systematic study indicated two main reasons for $M 2$ transitions with hindrance factors orders of magnitude larger than the single-particle estimates. For highly deformed heavy nuclei the hindrance factor could be explained by K-forbiddenness or by an asymptotic selection rule. While for light nuclei it was mainly due to isobaric spin effects. In the middle mass region it was proposed that both effects contribute.

\section{124.4-keV state}

A partial level scheme including the $124.4-\mathrm{keV}$ state and the related transitions is given in Fig. 8. The spin-parity of this state was proposed to be $1^{+}$due to direct $\beta$ feeding of $4 \%$ and $8 \%$ found in Refs. $[9,10]$, respectively. However, the 
position of this level was not clear and it was placed high in excitation energy. High-spin studies placed the level directly connected to the ground state with suggested spin values of $(2,3)$ in Refs. [12,13]. A more recent $\beta^{+} /$EC-decay work in Ref. [11] corroborates the position of the $124.4-\mathrm{keV}$ state but they assign a spin value of 1 based on the direct $\beta$ feeding of $0.93(44) \%$, leading to a $\log f t$ value of 6.12 .

The measured values of the conversion coefficients for the $124.4-\mathrm{keV}$ transition are $\alpha_{K}(\exp )=0.069(17)$ and $\alpha_{\mathrm{LM}}(\exp )=0.010(3)$ to be compared with the calculated values for $E 1 / M 1 / E 2$ multipolarities being $0.039 / 0.056 / 0.34$ for $\alpha_{K}\left(\right.$ th) and $0.00492 / 0.00727 / 0.053$ for $\alpha_{\mathrm{LM}}($ th), respectively. These values indicate a predominant $M 1$ character with $|\delta|=0.2(2)$ for this transition as shown in Fig. 5 and Table II.

The M1 multipolarity for the $124.4-\mathrm{keV}$ transition indicates positive parity for this state with possible spin values of $(0,1,2)$. Considering that the $\gamma$-ray transition intensities measured in our work are similar to those of Ref. [11], and taking into account their $\log f t$ value for this state, 6.12, the 0 and 2 spin values are excluded. Using the same arguments discussed previously for the ground state we adopt a $1^{+}$spin-parity for this state.

\section{131.7-keV state}

This state was observed both in fusion evaporation and in $\beta$-decay studies. It was tentatively assigned spin-parity of $\left(2^{-}\right)$ in Ref. [12] as part of a negative-parity band based on the $1^{-}$101.2-keV state. This level was removed from the band structure in the recent study reported in Ref. [16]. The $\left(2^{-}\right)$ assignment is consistent with the nonobservation of $\beta$ feeding to this level as deduced in Ref. [11]. A partial level scheme with the transitions involved in this discussion is shown in Fig. 7.

The only transition deexciting this level is the 30.4-keV transition. In this work, the $\alpha_{\mathrm{LM}}(\mathrm{exp})$ conversion coefficient was determined to be 5.1(13), see Table II. The comparison with theoretical values suggests $M 1+E 2$ character as they are $\alpha_{\mathrm{LM}}($ th $)=0.397 / 21.47$ for $M 1 / E 2$ multipolarities. Since this transition connects the $131.7-\mathrm{keV}$ state with the $3^{-} 101.2-\mathrm{keV}$ isomeric state, an $2^{-}$is the most likely spin-parity for the former, in agreement with the result of Refs. [11,12].

The $131.7-\mathrm{keV}$ level is fed by the $178.6-\mathrm{keV}$ transition which deexcites the well-known $1^{+}$state at 310.3(2) $\mathrm{keV}$ as shown in Fig. 8. Reference [11] indicated that there is a $\gamma$-ray transition of $177.2 \mathrm{keV}$ with a factor 19 lower in intensity. Thus, we assign the conversion electrons measured with the $4 \mathrm{~B} / 8 / 85$ miniorange configuration only to the $178.6-\mathrm{keV}$ transition. The resulting conversion coefficient is $\alpha_{K}(\exp )=0.013(3)$, to be compared with the calculated values for $E 1 / M 1 / E 2$ of $0.01351 / 0.02158 / 0.08999$. This very low value of the $\alpha_{K}$ (exp) justifies the assignment of $E 1$ to the $178.6-\mathrm{keV}$ transition. This result confirms the spin-parity assignment of $2^{-}$for the $131.7-\mathrm{keV}$ state.

It is interesting to indicate that the deexcitation scheme of the $131.7-\mathrm{keV}\left(2^{-}\right) \rightarrow 101.2-\mathrm{keV}\left(3^{-}\right) \rightarrow 0\left(1^{+}\right)$with a retarded $M 2$ transition is similar but inverted with respect to the one observed in the case of ${ }^{76} \mathrm{Br}: 102.6-\mathrm{keV}(4)^{+} \rightarrow$ $45.47-\mathrm{keV}(2)^{-} \rightarrow 0-\mathrm{keV}\left(1^{-}\right)$where a $57-\mathrm{keV}$ transition of
M2 character is found with a half-life of $1.31 \mathrm{~s}$. However, the 101.2-keV transition in ${ }^{72} \mathrm{Br}$ is clearly observed in $\beta$ decay while the $57.2-\mathrm{keV}$ in ${ }^{76} \mathrm{Br}$ is not.

\section{162.8-keV state}

$\mathrm{A} 1^{+}$spin-parity was assigned to this state in the very first $\beta$-decay study in Ref. [10] where $10 \%$ apparent $\beta$ feeding was estimated from the only six transitions assigned to ${ }^{72} \mathrm{Br}$ from the $\beta$ decay of ${ }^{72} \mathrm{Kr}$. This value was retained in the early highspin works $[12,13]$. However, a more recent $\beta^{+} /$EC study [11] that includes more than 100 transitions proposed an upper limit to the $\beta$ feeding of $0.25 \%$ and a $\log f t$ value of 6.72 , thus leading the authors to exclude this assignment although no alternative spin-parity was proposed.

The spin-parity of the $162.8-\mathrm{keV}$ level has been studied by two transitions of $162.8(2)$ and $38.4(2) \mathrm{keV}$ deexciting the state and three transitions feeding the state from the wellknown $1^{+}$states at 310.3(2), 415.5(4), and 577.2(4) keV. A simplified level scheme involving these transitions is shown in Fig. 8. The measured conversion coefficient for the 162.8$\mathrm{keV}$ transition indicates $M 1+E 2$ character with $|\delta| \approx 0.6$ as indicated in Table II and shown graphically in Fig. 5 .

The 38.4-keV transition deexciting the $162.8-\mathrm{keV}$ state to the $124.4-\mathrm{keV}$ one has a conversion coefficient of $\alpha_{K}(\exp )=$ 10 (3), between the theoretical values for $M 1$ and $E 2$ multipolarities of $\alpha_{K}(\mathrm{th})=1.479$ and 20.48 with a $|\delta|=0.9_{-0.26}^{+0.34}$. Therefore we propose an $M 1+E 2$ multipolarity for this transition.

These findings suggest that the parity of the $162.8-\mathrm{keV}$ state is positive. This assignment is corroborated by the $M 1$ character found for the $147.5-\mathrm{keV}$ transition feeding this state from the $1^{+} 310.3-\mathrm{keV}$ state and for the $414.7-\mathrm{keV}$ transition from the $1^{+}$state at $577.2(4) \mathrm{keV}$ and the $E 2$ character of the $252.7-\mathrm{keV}$ transition from the $1^{+}$state at $415.5(4) \mathrm{keV}$.

All these results consistently suggest that the most likely spin-parity for the $162.8-\mathrm{keV}$ level is either $0^{+}$or $2^{+}$. The $E 2$ component of the multipolarity of $38.4-$ and $162.8-\mathrm{keV}$ transitions excludes the $0^{+}$assignment. Thus this state is conclusively proposed to have a spin-parity of $2^{+}$.

\section{310.3-keV state}

This state has been observed both in fusion-evaporation and $\beta$-decay studies. Due to the strong $\beta$ feeding, a $1^{+}$assignment has been given to this state since the earliest works [9-13].

As mentioned, this level deexcites, among other transitions, via the $310.3-\mathrm{keV}$ transition to the ground state. The conversion coefficient measured is $\alpha_{K}(\exp )=0.0048(15)$ in agreement with the predicted value for an $M 1$ transition of 0.005351 . The possible mixing with $E 2$ multipolarity is at the level of the error bar, giving a mixing ratio $|\delta|=0^{+0.25}$. This suggests that the $310.3-\mathrm{keV}$ transition is predominantly $M 1$.

This state deexcites via other five transitions [11], two of them strong enough to study their conversion coefficients, see Table II. The 178.6-keV transition connecting this state with the $131.7-\mathrm{keV}$ state was found to be $E 1$, which is consistent with the $2^{-}$assignment for the $131.7-\mathrm{keV}$ state. The $310.3-\mathrm{keV}$ level is connected to the $162.8 \mathrm{-keV}$ state by the 
M1 147.5-keV transition reinforcing the $2^{+}$assignment to the 162.8-keV level.

\section{393.1-keV state}

This state has only been observed in the most recent $\beta^{+} /$EC-decay study of Piqueras et al. [11] and no spin assignment was given. The $\beta$ feeding was less than one per mille. Only two transitions have been identified deexciting this state to the $162.8-\mathrm{keV}$ and to the ground state, with energies of 230.5(2) and 393.1(4) keV, respectively.

The conversion coefficient measured for the low-intensity $393.1-\mathrm{keV}$ transition is $\alpha_{K}(\exp )=0.018(7)$ to be compared with the theoretical values of $0.011 / 0.019$ for $M 2 / E 3$ multipolarities, respectively. In a first instance we propose $E 3(M 2)$. However, one should keep in mind that the Weisskopf estimate for an $E 3$ transition corresponds to a partial half-life of 1.7 ms while for an $M 2$ is $\approx 120$ ns. In the work of Ref. [11] coincidence between the $393.1-\mathrm{keV}$ and other $\gamma$-ray lines (time window of $\approx 500 \mathrm{~ns}$ ) from higher excited states were observed limiting the half-life of this state to a maximum of a few hundred ns. We propose the transition to be $M 2(E 3)$ compatible with our results within the error bars. This implies a set of most likely spin-parity values of $(3,4)^{-}$for this level.

Regarding the $230.5-\mathrm{keV}$ transition, the $\gamma$-ray line is weak ( 0.57 per 100 decays) and the electron energy lies in a region with very low electron transmission for both the $4 \mathrm{~B} / 8 / 85$ and $6 \mathrm{~A} / 8 / 45$ miniorange configurations. It is difficult to obtain a reliable value for the conversion coefficient due to low number of counts in the region of interest. Therefore we assign an $E 1$ multipolarity for this transition which corroborates the change in parity between the 393.1 - and the $162.8-\mathrm{keV}$ levels.

Taking into account these findings, we are not able to definitely fix the spin-parity of this state although we favor $(3)^{-}$.

\section{8. $398.9-\mathrm{keV}$ state}

This level was first observed in high-spin studies using the reaction ${ }^{58} \mathrm{Ni}\left({ }^{16} \mathrm{O}, n p\right){ }^{72} \mathrm{Br}$ [12] and was corroborated in Ref. [13] as the $\left(2^{+}\right)$member of the positive-parity band. This value was retained in later fusion-evaporation studies $[14,15]$. The $\beta^{+} /$EC-decay study of Piqueras et al. [11] indicated no $\beta$ feeding to this state, so their result was compatible with the previous $\left(2^{+}\right)$assignment. However, in the most recent high-spin study [16], this level became the head of the negative-parity band 4, following the CNS calculation as formulated in Ref. [19] with three protons and four neutrons in the $g_{9 / 2}$ orbital. They proposed for this state $J^{\pi}=\left(2^{-}\right)$. The parenthesis in the spin assignment was due to the uncertainty in the spin-parity assignment for the ground state.

The 398.9-keV transition connecting with the $1^{+}$ground state is seen with low intensity in our $\beta$ decay of ${ }^{72} \mathrm{Kr}$ as in previous one [11]. The conversion coefficient has been measured giving an upper limit of $\alpha_{K}(\exp ) \leqslant$ $0.002(2)$, to be compared with the theoretical values of $0.001424 / 0.00292 / 0.0054$ for $E 1 / M 1 / E 2$ multipolarities. This result is not conclusive allowing for $E 1$ and $M 1$ multipolarities for this transition.
The half-life of the 398.9-keV state has been measured to be $101(20)$ ps $[13,21]$. We can determine the single-particle transition half lives to be $3.8 \mathrm{e}-3 / 0.21 / 1900$ ps for $E 1 / M 1 / E 2$ multipolarities, taking into account the recommended upper limits for $T_{1 / 2}(\exp ) / T_{1 / 2}$ (W.u.) [45] being $0.01 / 3 / 300$ for $E 1 / M 1 / E 2$ multipolarities. In our case these ratios are 26000/0.002/19. Considering the limit on the conversion coefficient and the upper limits for the $T_{1 / 2}$ single-particle estimates, our result favors an $M 1$ multipolarity, although a very retarded $E 1$ transition cannot be fully discarded. Therefore, we suggest that this transition is $(M 1)$ precluding a firm assignment of parity for the $398.9-\mathrm{keV}$ state. Thus the spinparity of the $398.9-\mathrm{keV}$ state is assigned to be $2^{(+)}$.

\section{9. $415.5-\mathrm{keV}$ state}

The transition deexciting the $415.5-\mathrm{keV}$ state to the ground state turns out to be a doublet according to Ref. [11]. The 414.7-keV transition connecting the state at 576.2(4) keV with the one at 162.8(2) keV (see the partial level scheme in Fig. 8) can be resolved neither in the HPGe spectrum nor in the $\mathrm{Si}(\mathrm{Li})$ spectrum from the 415.5-keV transition deexciting the 415.5$\mathrm{keV}$ level. So we have to evaluate together the conversion coefficient of the D415 transition.

The experimental conversion coefficient of $\alpha_{K}(\exp )=$ $0.0023(3)$ is obtained as the weighted average of the values from two different miniorange configurations, $6 \mathrm{~A} / 8 / 45$ and $6 \mathrm{~A} / 8 / 60$. The theoretical values of the conversion coefficients for $E 1 / M 1 / E 2$ multipolarities are $0.00128 / 0.002649 / 0.004725$. Our result is in good agreement with the theoretical value for $M 1$ multipolarity. This agrees with previous works such as Refs. [9-11], where they found that the $415.5-\mathrm{keV}$ state is strongly fed in the $\beta^{+} / \mathrm{EC}$ decay of ${ }^{72} \mathrm{Kr}$ indicating that the most probable spin-parity is $1^{+}$ confirming the $M 1$ multipolarity for the $415.5-\mathrm{keV}$ transition.

The 414.7-keV transition of the doublet connects the $1^{+}$ $577.2-\mathrm{keV}$ state with the $162.8 \mathrm{-keV}$ one. The $M 1$ character of this transition also corroborates the $2^{+}$assignment for the $162.8-\mathrm{keV}$ state.

\section{0. $546.4-\mathrm{keV}$ state}

This level has only been observed in the work of Piqueras et al. [11] with a $\beta$ feeding lower than $0.15 \%$ and no spin assignment. Only two transitions of $147.5(2)$ and 235.8(2) $\mathrm{keV}$ were established to deexcite this level to the 398.9- and 310.3-keV states, see Fig. 9.

The K-shell conversion electrons of the $235.8-\mathrm{keV}$ transition are at the very low efficiency limit of the two miniorange configurations, $4 \mathrm{~B} / 8 / 85$ and $6 \mathrm{~A} / 8 / 45$. The corresponding conversion coefficients are $\alpha_{K}(\exp )=$ $0.05(3)$ and $0.07(4)$. Being the weighted mean value $\alpha_{K}(\exp )=0.05(2)$. This value can be compared with the theoretical values for $E 1 / M 1 / E 2 / M 2 / E 3$ multipolarities of $0.006085 / 0.01066 / 0.03324 / 0.0555 / 0.1548$, respectively. The conversion coefficient favors $M 2$ multipolarity for this transition although the option of $E 2$ is possible within the error bars. The single-particle estimates for $E 2$ gives a halflife for the state of $37 \mathrm{~ns}$ meanwhile for $M 2$ provides 2.1 $\mu \mathrm{s}$. Considering that this transition of $235.8 \mathrm{-keV}$ has been 


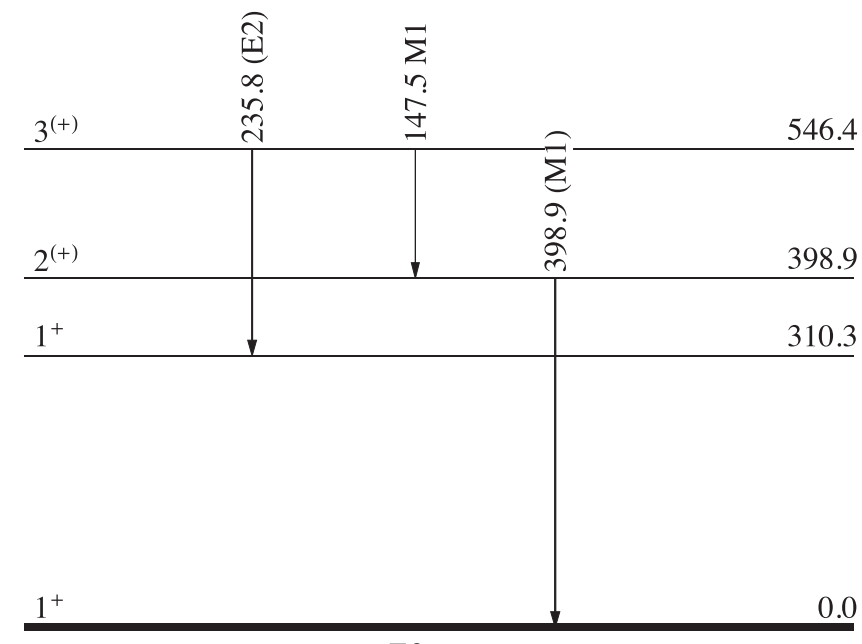

${ }^{72} \mathrm{Br}$

FIG. 9. Levels and transitions involved in the discussion of the spin-parity assignment of the $398.9-\mathrm{keV}$ state in ${ }^{72} \mathrm{Br}$.

seen in coincidence in previous works [11] we propose an E2 multipolarity for this transition but $M 2$ cannot be fully rejected.

Therefore, the most favorable spin-parity value for this level is $3^{(+)}$. This result is consistent with the $M 1147.5-\mathrm{keV}$ transition connecting this state with the $398.9-\mathrm{keV} 2^{(+)}$state.

\section{576.2- and 577.2-keV states}

Initially, these two states at 576.2(4) $\mathrm{keV}$ and 577.2(4) keV were considered as one in Refs. [9,10]. However, in the higher statistics and resolution study of Ref. [11] it was observed as two independent levels with $\beta$ feeding of 1.44 and $13.06 \%$ giving $\log f t$ values of 5.74 and 4.78 , respectively. These strongly fed levels have not been observed in any of the fusion-evaporation studies $[12,13,15,16]$.

The partial level schemes including these states and the transitions involved in the discussion are shown in Figs. 8 and 9. An apparent single $\gamma$-ray line is found at $577.2-\mathrm{keV}$ which is broader than the rest so we force the fit to two Gaussian components placed at energies of 576.2 and $577.2 \mathrm{keV}$. We find a factor of 7.9(11) between the relative intensities of the two transitions, not far from the value of 5.5(7) found in the literature based on $\gamma-\gamma$ coincidence analysis [11]. The conversion electrons were measured using two miniorange configurations, $6 \mathrm{~A} / 8 / 45$ and $6 \mathrm{~A} / 8 / 60$, giving consistent values of $\alpha_{K}(\exp )=0.0014(5)$ and $0.0010(4)$. The average value of $0.0012(2)$ is in perfect agreement with the theoretical value of $M 1$ multipolarity as the expected values for $E 1 / M 1 / E 2$ multipolarities are $\alpha_{K}($ th $)=0.000568 / 0.001237 / 0.001718$. This agreement indicates that both transitions in the doublet should be $M 1$. This is in agreement with Ref. [11], where the spin-parities of both states were assigned to be $1^{+}$.

\section{Level scheme of ${ }^{72} \mathrm{Br}$}

The partial level scheme for all the low-lying levels studied in the ${ }^{72} \mathrm{Br}$ nucleus is shown in Fig. 10. The multipolarities of
15 transitions have been firmly determined with 4 being of mixed character. The 235.8- and 398.9-keV transitions have only been given tentative multipolarity assignments. As a result, the spin-parities of 9 states in ${ }^{72} \mathrm{Br}$ are now fixed while the 398.9- and 546.4-keV states remain with fixed spin and probable parity. The state at $393.1-\mathrm{keV}$ has a fixed negative parity with a probable spin of 3 .

\section{COMPARISON WITH THEORETICAL CALCULATIONS}

A number of different theoretical approaches are available to describe the properties of nuclei in the $A \approx 70-80$ region of the chart of nuclides.

Calculations for the odd-odd ${ }^{72} \mathrm{Br}$ nucleus have been performed within the framework of the two quasiparticle (2qp) + rotor model [48]. In this simple model, the 2qp intrinsic states describe the valence proton and neutron configurations and represent the bandheads of associated rotational bands. They are obtained from axially deformed Hartree-Fock meanfield calculations with the SLy4 Skyrme interaction [49] and include pairing correlations in the BCS approximation.

Figure 11 shows the level scheme obtained in this work in comparison with those obtained with different theoretical approaches. On the left-hand side, the results from 2qp+rotor model are shown for both prolate $\left(\beta_{2}=0.12\right)$ and oblate $\left(\beta_{2}=-0.18\right)$ deformations that produce energy minima in the HF+BCS(SLy4) calculation [50]. In the prolate case the lowest energy corresponds to the $\pi[301] 3 / 2 \otimes \nu[301] 3 / 2$ configuration that couple to form $0^{+}$and $3^{+}$states. The magnetic moment has been calculated using the formula of J. Kern and G. L. Struble [51] with the parameters obtained for ${ }^{71} \mathrm{Br}$ and ${ }^{73} \mathrm{Kr}$. The magnetic moment obtained for this configuration is $0.88 \mu_{N}$. According to the Gallagher-Moszkowski rules [52] the ground-state bandhead is $3^{+}$and the excited bandhead corresponds to $0^{+}$total angular momentum. As no $p-n$ residual interactions are included these two bandheads appear to be degenerate in this calculation as shown in Fig. 11. In the oblate case, the ground state involves the odd proton and odd neutron deformed orbitals $\pi[310] 1 / 2 \otimes v[310] 1 / 2$, that couple to form $1^{+}$and $0^{+}$states. According to the GallagherMoszkowski rules [52], the ground state becomes $1^{+}$. The calculated magnetic moment for this configuration is $0.57 \mu_{N}$.

As can be seen in Fig. 11, the oblate calculation reproduces better the experimental level scheme. The calculated excited $1^{+}$states at 101.3 and $108.9 \mathrm{keV}$ energy in the oblate case correspond to $\pi[301] 3 / 2 \otimes v[310] 1 / 2$ and $\pi[310] 1 / 2 \otimes$ $v[301] 3 / 2$ configurations and correlate well with the measured excited state at $124 \mathrm{keV}$. The states at 322.6 and $532.8 \mathrm{keV}$ correspond to rotational states built on top of the 2qp ground-state, $\pi[310] 1 / 2 \otimes v[310] 1 / 2$ and $\pi[301] 3 / 2 \otimes$ $\nu[301] 3 / 2$ configurations. The state at $889 \mathrm{keV}$ is based on the 2qp $\pi[321] 1 / 2 \otimes v[310] 1 / 2$ configuration.

Checking the spin-parity of neighboring bromine isotopes, ${ }^{71} \mathrm{Br}$ is proposed to have a $(5 / 2)^{-}$ground state [53] while ${ }^{73} \mathrm{Br}$ is firmly assigned $1 / 2^{-}$[54]. The structures of these states are discussed in Ref. [20] to be both oblate. While the structure of

${ }^{71} \mathrm{Br}$ ground state is rather complex, a dominant single-particle [310]1/2 orbital is assigned for the unpaired proton of ${ }^{73} \mathrm{Br}$ ground state. This is in line with the configuration proposed 


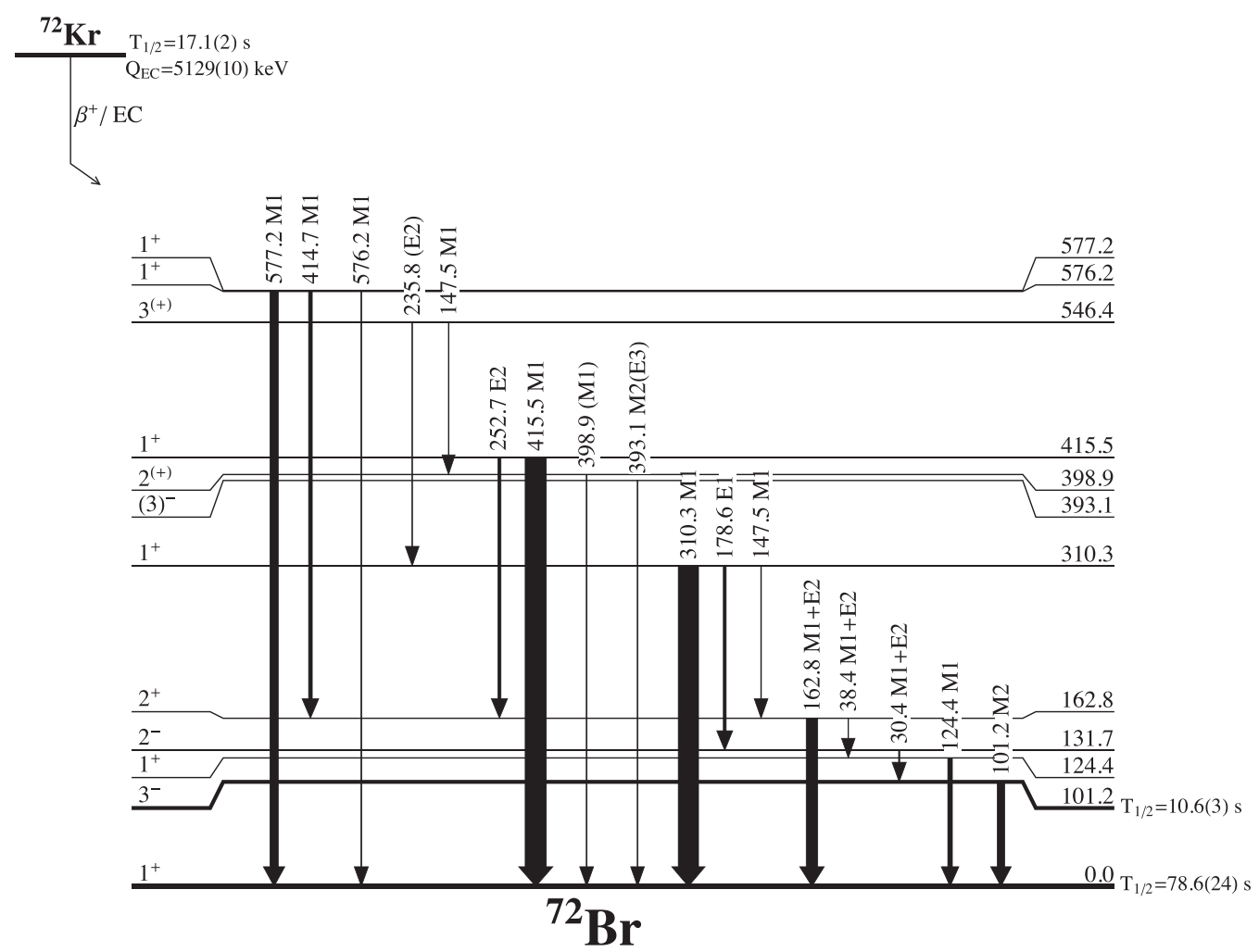

FIG. 10. Low-energy region of the level scheme of ${ }^{72} \mathrm{Br}$ including the multipolarities and spin-parities of levels obtained through the conversion coefficients measured in this work. The level and transition energies given are those obtained in this work. The parent half-life and QEC values are taken from Ref. [21]. The thickness of transition arrows follows the $\gamma$ transition intensities from this work given in Table I.

for the ${ }^{72} \mathrm{Br}$ ground state in our $\mathrm{HF}+\mathrm{BCS}(\mathrm{SLy} 4)$ calculations for the oblate case as $\pi[310] 1 / 2 \otimes v[310] 1 / 2$. The calculated magnetic moment of $0.57 \mu_{N}$ reproduces well the experimental value of $0.55(21) \mu_{N}[20]$.

Concerning the isomeric state at $101.2-\mathrm{keV}$ energy, ${ }^{72 m} \mathrm{Br}$, the magnetic moment was determined to be $1.3_{-0.6}^{+10} \mu_{N}$ [55] with a large background. Therefore, a lower limit for the magnetic moment of $\mu>0.7 \mu_{N}$ was determined and it is this value which has passed to the literature [21]. A similar magnetic moment was found for the ${ }^{74 m} \mathrm{Br}$ state in Ref. [20] to be $\mu=1.68(18) \mu_{N}$. This value of the magnetic moment was compatible with both a single-particle configuration of $\pi[301] 3 / 2 \otimes \nu[422] 5 / 2$ giving a $4^{-}$spin and a magnetic moment of $\mu=1.71 \mu_{N}$ and the $\pi[431] 3 / 2 \otimes \nu[422] 5 / 2$
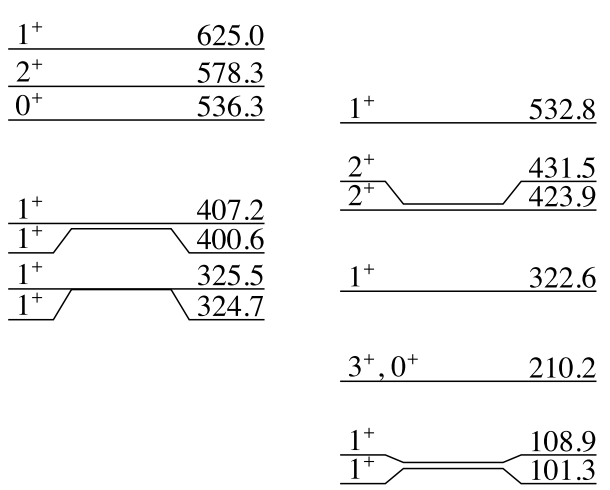

$3^{+}, 0^{+}$ 0.0
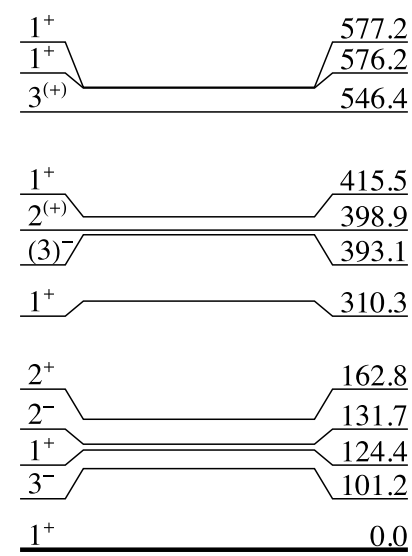

Experiment
$1^{+}$

$1^{-}$

$1^{+}$ 448.5

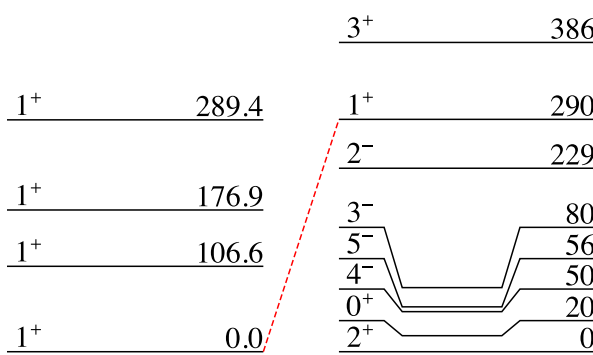

Shell Model jun45.Inps

$1^{+}$states
Shell Model jun45.Inps Oblate

FIG. 11. Partial level scheme of ${ }^{72} \mathrm{Br}$. Comparison of the our experimental and theoretical results. Theoretical calculations were done using the mean-field HF+BCS approach with the SLy4 Skyrme-type interaction and large-scale shell-model calculations using the jun45.lnps interaction. 

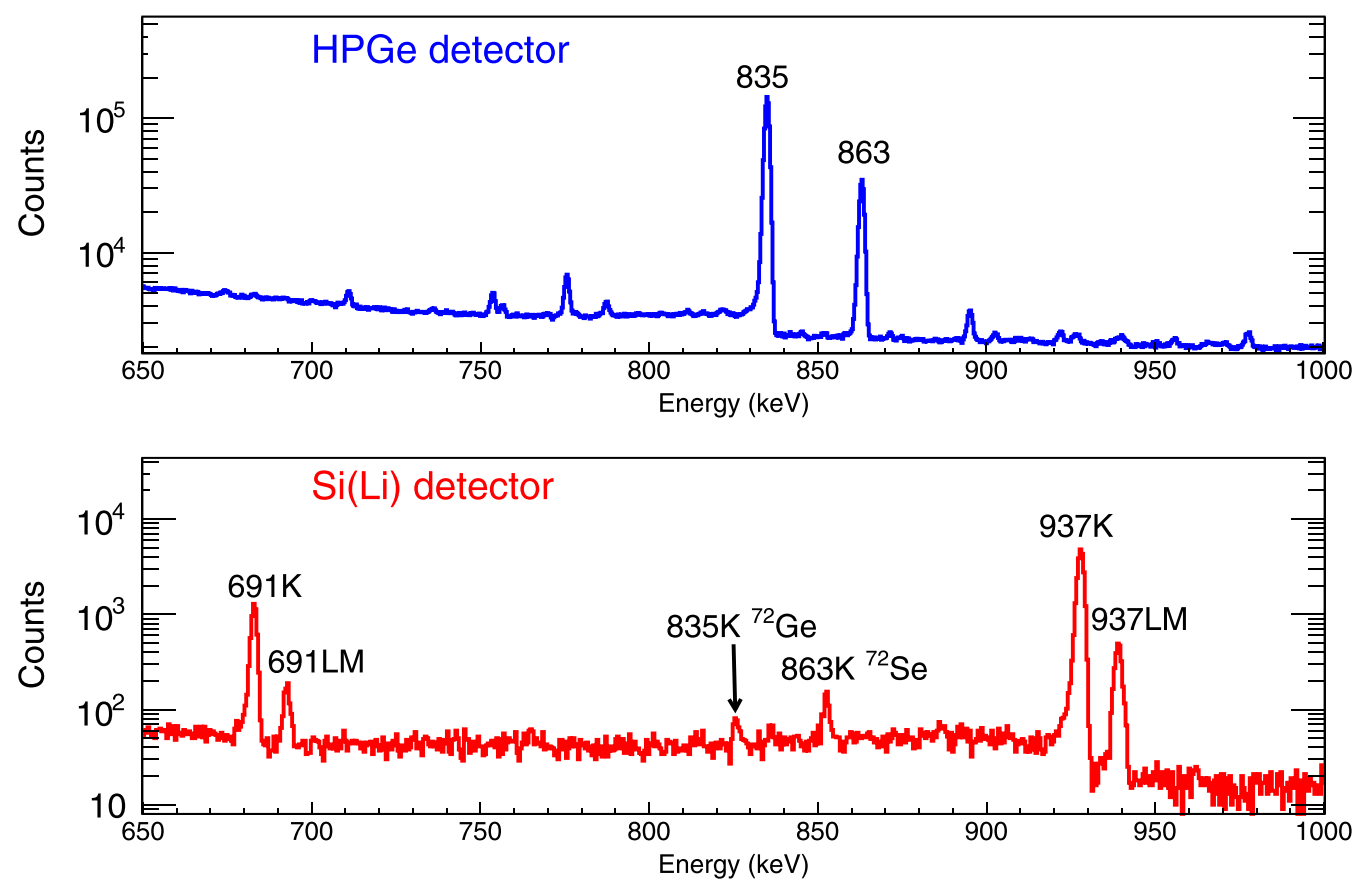

FIG. 12. HPGe and $\mathrm{Si}(\mathrm{Li})$ spectra taken using the $6 \mathrm{~A} / 8 / 60$ miniorange configuration. Two well-known $E 2$ transitions in the descendants ${ }^{72} \mathrm{Se}$ and ${ }^{72} \mathrm{Ge}$ are identified in the HPGe spectrum and electron transitions associated with two $E 0$ transitions in the same nuclei are seen in the $\mathrm{Si}(\mathrm{Li})$ spectrum.

configuration corresponding to a $4^{+}$spin and an estimated $\mu=1.77 \mu_{N}$.

In our case, we assume for the $3^{-}{ }^{72 m} \mathrm{Br}$ state the same single-particle orbital for the proton than in the ground state, $\pi[301] 3 / 2$, and for the neutron we propose the lower [431]3/2 deformed orbital. In our mean-field calculations this configuration is prolate and its calculated magnetic moment amounts to $1.40 \mu_{N}$ which reproduces well the experimental value of $1.3_{-0.6}^{+10} \mu_{N}$ [55].

This drastic change in deformation in only $101 \mathrm{keV}$ between the oblate ${ }^{72} \mathrm{Br}$ ground state and the prolate ${ }^{72 m} \mathrm{Br}$ isomeric state could explain the large hindrance factor of $>60000$ for the $M 2$ transition connecting both states.

Our experimental results are also compared with largescale shell-model calculations. They have been performed in the valence space encompassing the orbits $1 p_{3 / 2}, 0 \mathrm{f}_{5 / 2}, 1 p_{1 / 2}$, $0 g_{9 / 2}$, and $1 d_{5 / 2}$ i.e., with a ${ }^{56} \mathrm{Ni}$ core. The effective interaction is based in the JUN45 of Ref. [56], supplemented with the matrix elements involving the $1 d_{5 / 2}$ taken from the LNPS interaction, that we dub JUN45.Inps in Ref. [57]. The dimension of the full space calculation is beyond reach, and we have truncated it allowing up to $4 \mathrm{p}-4 \mathrm{~h}$ jumps across $N=Z=40$. Full convergence is not guaranteed. The results of these calculations are shown on the right-hand side of Fig. 11. They produce a ground state which has a spin-parity $2^{+}$and the first $1^{+}$state appears at $290 \mathrm{keV}$, at difference of the experimental results. However, this is not surprising because of the high level density and the limitations of the calculation in an oddodd nucleus. On the positive side, one can notice the presence of negative-parity states at low-excitation energies as observed experimentally in contrast with the result of HF+BCS (SLy4) calculations. On the middle right-hand side column the distri- bution of $1^{+}$states for this calculation is given. Five $1^{+}$levels up to $610 \mathrm{keV}$ of excitation energy are predicted in agreement with our experimental results. The sequence of negative-parity states compare reasonably well with the scarce experimental information.

From the comparison with $\mathrm{HF}+\mathrm{BCS}$ (SLy4) calculations an oblate deformation is suggested for the ${ }^{72} \mathrm{Br}$ ground state with a $1^{+}$spin-parity. The relevant direct $\beta$ feeding from the ${ }^{72} \mathrm{Kr} 0^{+}$ground state to the ${ }^{72} \mathrm{Br}$ ground state deduced in several experiments [9-11] supports the $1^{+}$assignment indicating a similar deformation for the parent and daughter ground states. These two facts suggest oblate deformation for both ${ }^{72} \mathrm{Kr}$ and ${ }^{72} \mathrm{Br}$ ground states. This conclusion is supported by different probes indicating an oblate character for the ${ }^{72} \mathrm{Kr}$ ground state [23-26].

\section{SEARCH FOR EO TRANSITIONS}

We searched for $E 0$ transitions in the decay of interest. No such transitions were identified belonging to ${ }^{72} \mathrm{Br}$. However, intense $E 0$ transitions were found in our measurements with miniorange configurations $6 \mathrm{~A} / 8 / 45$ and $6 \mathrm{~A} / 8 / 60$ belonging to $A=72$ isobars.

Figure 12 shows the $\gamma$-ray and conversion electron spectra in the energy range between 650 and $1000 \mathrm{keV}$ measured with the $6 \mathrm{~A} / 8 / 60$ miniorange configuration. The $E 0$ transitions at 691 and $937 \mathrm{keV}$ are clearly visible and assigned to the lowest $0^{+} \rightarrow 0^{+}$transitions in ${ }^{72} \mathrm{Ge}$ and ${ }^{72} \mathrm{Se}$, respectively. As expected, no corresponding $\gamma$-ray transition is seen. Additionally, the conversion electron lines from the $863-\mathrm{keV}$ $E 2$ transition in ${ }^{72} \mathrm{Se}$ and the $835-\mathrm{keV} E 2$ transition in ${ }^{72} \mathrm{Ge}$ are also visible. In the $\gamma$-ray spectrum, these lines dominate 

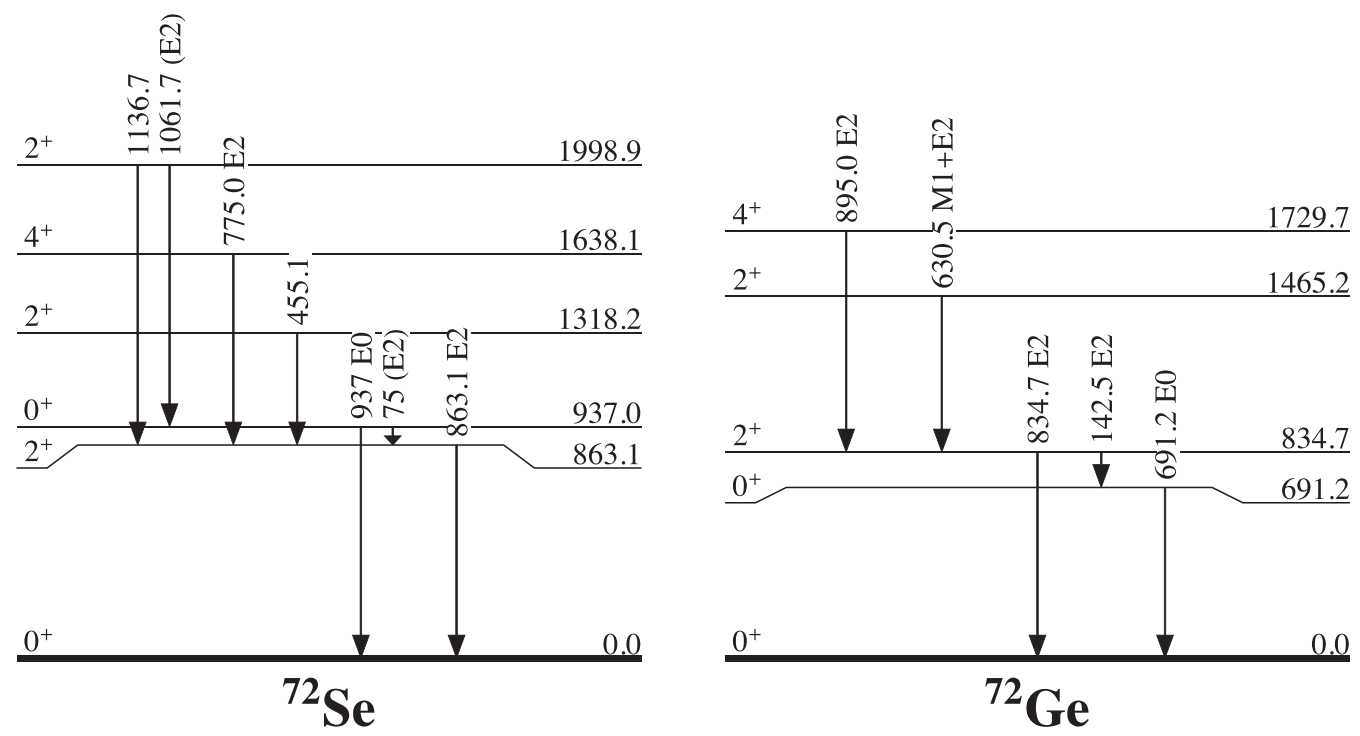

FIG. 13. Partial level scheme of ${ }^{72} \mathrm{Se}$ (left) and ${ }^{72} \mathrm{Ge}$ (right) showing the deexcitation transitions relevant for our discussion and those whose intensity is given in Tables IV and III. Values for levels and transition energies are obtained in this work and those missing are taken from Ref. [21]. Spin-parities and transition mutipolarities are taken from Ref. [21].

this energy region. See the partial level schemes shown in Fig. 13.

The intensity of the E0 937-keV transition in ${ }^{72} \mathrm{Se}$ was deduced from comparison of the relative intensities of the 1061.7- and 1136.7-keV transitions feeding the 937- and 863$\mathrm{keV}$ states (see Fig. 13) in singles and $\gamma-\gamma$ coincidences gated by the $862-\mathrm{keV}$ transition [1]. The relative intensity of the $E 0$ transition with respect to the $862-\mathrm{keV}$ transition was determined to be 3.3(17)\%. In addition, they deduced the ratio for the two transitions deexciting the $937-\mathrm{keV}$ state to be $0.37(23)$ [1]. A more recent value of 0.41(8) have been obtained using the same methodology in Ref. [46].

The $E 0$ transition was directly measured in in-beam studies [58]. The feeding to the 862- and 937-keV states in the ${ }^{70} \mathrm{Ge}(\alpha, 2 n){ }^{72} \mathrm{Se}$ reaction used in Ref. [58] is different from the one obtained in the ${ }^{72} \mathrm{Br} \beta$ decay. Therefore, the relative intensities cannot be directly compared.

The $E 0$ transition of $691 \mathrm{keV}$ in ${ }^{72} \mathrm{Ge}$ was directly measured by the same group from ${ }^{72} \mathrm{Ga}[59]$ and ${ }^{72} \mathrm{As}$ [60] decays establishing a relative intensity with respect to the $834-\mathrm{keV}$ transition of $0.52(5)$ and $2.3(4) \%$, respectively. In spite of using the same $\mathrm{Si}(\mathrm{Li})$ electron spectrometer, in the case of the ${ }^{72}$ As decay the $\mathrm{K}$ component could not be resolved from the LM one. The total intensities of the 691- and 834-keV transitions were obtained from the electron spectrum assuming an E2 multipolarity for the 834-keV transition.

We followed the same analysis procedure that used in Refs. [59,60] and deduced the $\gamma$ intensity for the $E 2$ transitions from their conversion electrons. This is done to avoid the use of the $\gamma$-ray transition intensities since the HPGe detector

TABLE III. Results for the $\gamma$-ray and $E 0$ transition intensities compared with the most intense $E 2$ transition in the excitation scheme of ${ }^{72} \mathrm{Se}$. They belong to measurements performed using the $6 \mathrm{~A} / 8 / 60$ miniorange configuration but with two different tape-moving cycles $\left(T_{c}\right)$ : 33.6 and $100.8 \mathrm{~s}$. The upper part of the table shows the comparison of the relative $\gamma$-ray transition intensities with tabulated values. The lower part shows the relative intensity of the electron lines (K, LM and their sum) with respect to the most intense $E 2$ transition in ${ }^{72}$ Se. When two uncertainties are given, first component accounts for statistical plus systematic and the second for normalization.

\begin{tabular}{|c|c|c|c|c|c|}
\hline $\begin{array}{l}E_{\gamma}(\mathrm{keV}) \\
\text { (this work) }\end{array}$ & \multicolumn{2}{|c|}{$I_{\gamma}$} & $\begin{array}{c}I_{\gamma} \\
\text { Adopted }\end{array}$ & $\begin{array}{c}E_{\gamma}(\mathrm{keV}) \\
{[21]}\end{array}$ & $\begin{array}{c}I_{\gamma} \\
{[21]}\end{array}$ \\
\hline $455.1(4)$ & $16.5(17)(16)$ & $17.2(17)(17)$ & $16.9(17)$ & $454.7(1)$ & $18.7(11)$ \\
\hline \multirow[t]{2}{*}{$775.0(4)$} & $8.7(9)(9)$ & $9.0(9)(9)$ & $8.9(9)$ & 774.73(17) & $10.1(6)$ \\
\hline & \multicolumn{2}{|c|}{$I(\gamma+\mathrm{EC})$} & $I(\gamma+\mathrm{EC})$ & $I(\gamma+\mathrm{EC})$ & \\
\hline 863.1 & $100(22)$ & $100(24)$ & $100(16)$ & $100 *$ & \\
\hline $937 \mathrm{~K}$ & $4.5(9)(10)$ & $4.8(10)(12)$ & $4.7(10)$ & & \\
\hline 937LM & $0.54(11)(12)$ & $0.59(12)(14)$ & $0.56(14)$ & & \\
\hline 937 & $5.0(14)(15)$ & $5.4(16)(19)$ & $5.2(16)$ & $3.3(17)$ & \\
\hline
\end{tabular}


TABLE IV. Results for the $\gamma$-ray and $E 0$ transition intensities compared with the most intense $E 2$ transition in the excitation scheme of ${ }^{72} \mathrm{Ge}$. They belong to two measurements performed using the 6A/8/60 miniorange configuration with different tape-moving cycles $\left(T_{c}\right)$ : 33.6 and $100.8 \mathrm{~s}$. The upper part shows the comparison of the relative $\gamma$-ray transition intensities with tabulated values from ${ }^{72} \mathrm{Ga}$ and ${ }^{72} \mathrm{Ar}$ decays. The lower part shows the relative intensity of the electron lines (K, LM and their sum) with respect to the most intense $E 2$ transition in ${ }^{72} \mathrm{Ge}$. When two uncertainties are given, first component accounts for statistical plus systematic and the second for normalization.

\begin{tabular}{|c|c|c|c|c|c|c|}
\hline $\begin{array}{l}E_{\gamma}(\mathrm{keV}) \\
\text { (this work) }\end{array}$ & \multicolumn{2}{|c|}{$I_{\gamma}$} & $\begin{array}{c}I_{\gamma} \\
\text { Adopted }\end{array}$ & $\begin{array}{c}E_{\gamma}(\mathrm{keV}) \\
{[21]}\end{array}$ & $\begin{array}{c}I_{\gamma}[21] \\
\text { from }{ }^{72} \text { Ga decay }\end{array}$ & $\begin{array}{c}I_{\gamma}[21] \\
\text { from }{ }^{72} \text { As decay }\end{array}$ \\
\hline $630.5(4)$ & $6.0(6)(6)$ & $7.1(7)(7)$ & $6.5(6)$ & $629.95(3)$ & $27.38(4)$ & $9.96(17)$ \\
\hline \multirow[t]{2}{*}{$895.0(4)$} & $1.22(13)(12)$ & $1.29(16)(13)$ & $1.25(18)$ & $894.26(4)$ & $10.619(14)$ & $0.975(15)$ \\
\hline & \multicolumn{2}{|c|}{$I(\gamma+\mathrm{EC})$} & $I(\gamma+\mathrm{EC})$ & $I(\gamma+\mathrm{EC})[21]$ & $I(\gamma+\mathrm{EC})[21]$ & \\
\hline 834.7 & $100(26)$ & $100(49)$ & $100(23)$ & 100 & 100 & \\
\hline $691.2 \mathrm{~K}$ & $0.57(11)(15)$ & $0.59(12)(29)$ & $0.58(16)$ & & & \\
\hline 691.2LM & $0.069(14)(18)$ & $0.063(14)(30)$ & $0.066(18)$ & & & \\
\hline 691.2 & $0.64(18)(23)$ & $0.66(20)(45)$ & $0.65(24)$ & $0.52(5)$ & $2.0(4)$ & \\
\hline
\end{tabular}

could detect $\gamma$ rays from previous samples on the tape. The detector was shielded with lead bricks but the system was too compact and the half-life of the ${ }^{72} \mathrm{Br}$ and ${ }^{72} \mathrm{As}$ decays long.

Table III shows the results obtained for the ${ }^{72}$ Se transitions. The $\gamma$-ray transition intensities are given in the upper part on column 4 , and they agree nicely with the tabulated values given in column 5 within the error bars. Then, the lower part shows the relative intensity of the $937-\mathrm{keV} E 0$ transition as compared to the total intensity for the $863-\mathrm{keV} E 2$ transition. The obtained relative intensity of 5.2(16) agrees with the tabulated value of 3.3(17) [21] which was obtained indirectly via comparison of $\gamma$-ray singles and $\gamma-\gamma$ coincidences by Hamilton et al. [1]. The present value is the first direct measurement of the relative intensity of this $E 0$ transition with respect to the $863-\mathrm{keV} E 2$ transition fed via $\beta$ decay, and is compatible with the one deduced from $\gamma$-ray spectroscopy although it is slightly higher and more precise. In the same line, we use the conversion electron of the $75-\mathrm{keV}$ transition to deduce the ratio of intensities of the two transitions deexciting the 937-keV $0^{+}$state. The relative total intensities of the $E 0$ and $E 2$ transitions deexciting the $937-\mathrm{keV}$ state is $0.34(10)$. This value obtained from conversion electrons agrees with those obtained from $\gamma-\gamma$ coincidences $[1,46]$.

Table IV shows the results obtained for the ${ }^{72} \mathrm{Ge}$ transitions. The tabulated values in columns 5 and 6 are based on the measurements done by Rester et al. [59,60]. The $\gamma$-ray transition intensities are given in the upper part of Table IV, and they agree with the tabulated values for the ${ }^{72} \mathrm{As}$ decay and they are far from those of ${ }^{72} \mathrm{Ga}$ decay. The large difference of vapor pressure of $\mathrm{As}$ and $\mathrm{Ga}$ in the ISOLDE hot target explains why the main impurity of the incoming beam is of ${ }^{72}$ As.

The lower part of Table IV shows the relative intensity of the 691-keV E0 transition as compared to the total intensity for the $835-\mathrm{keV} E 2$ transition. We are surprised that the relative intensity obtained for the ${ }^{72} \mathrm{As}$ decay is similar to that given in Ref. [21] for the ${ }^{72} \mathrm{Ga}$ decay. Our result is a factor of 3 smaller than found in Ref. [60].
It is surprising that our relative intensities do not agree with the values provided in the data evaluation of Ref. [21] for the ${ }^{72}$ As decay, however, they do agree with the relative values for the ${ }^{72} \mathrm{Ga}$ decay. It is worth mentioning that although it is claimed in Refs. [59,60] an energy resolution of $5.5 \mathrm{keV}$ for the electron spectrometer of both measurements, in the study of ${ }^{72}$ As decay [60] the electron spectrum shows a much poorer resolution. Therefore, we propose for the $\beta$ decay of ${ }^{72}$ As to ${ }^{72} \mathrm{Ge}$ that the relative intensity of the $691-\mathrm{keV} \mathrm{E0}$ transition is $0.65(24) \%$ with respect to the $835-\mathrm{keV} E 2$ transition.

\section{SUMMARY AND CONCLUSION}

The conversion electron spectroscopy study of the $\beta^{+} / \mathrm{EC}$ decay of ${ }^{72} \mathrm{Kr}$ has been performed using a $\mathrm{Si}(\mathrm{Li})$ detector coupled to an electron spectrometer with 4 different miniorange configurations, thus optimising the electron transmission in different energy ranges up to $1 \mathrm{MeV}$. A HPGe detector has been used to measure the $\gamma$-ray intensities.

The deduced conversion coefficients have been used to determine the multipolarities of 17 transitions. A discussion of the spins and parities of the low-lying levels in ${ }^{72} \mathrm{Br}$ is presented.

The spin and parity of the ground state of ${ }^{72} \mathrm{Br}$ is definitely established as $1^{+}$from the $M 1$ multipolarity of the three transitions connecting the well-known $1^{+}$states with the ground state. This determination is relevant for the fusion-evaporation studies to firmly assign the spin-parities of the states in the rotational bands. One should notice that all these studies assumed that the ground state was $\left(3^{+}\right)$. Therefore, the various interpretations of the bands should be revisited.

The spin of the isomeric state is established to be $3^{-}$and the $M 2$ transition connecting with the ground state has the largest hindrance factor known for a radiative $M 2$ transition. The possible existence of a $\beta$-decay branch from this state, as occurs in a similar case in ${ }^{70} \mathrm{Cu}$, could contribute to explain the $5 \%$ feeding to the $4^{+}$state in ${ }^{72}$ Se which motivated the $3^{+}$ assignment to the ${ }^{72} \mathrm{Br}$ ground state in the past. 
The low-energy level scheme proposed in this work is compared with the truncated shell model using a ${ }^{56} \mathrm{Ni}$ core and $4 \mathrm{p}-4 \mathrm{~h}$ excitations and mean-field calculations using the SLy4 Skyrme force in BCS approximation. The comparison with the shell model case indicates the same number of $1^{+}$ states in the level scheme up to $600-\mathrm{keV}$ excitation energy. However, the energy spacing does not follow the experimental energy distribution. Our level scheme shows similarities with the mean-field calculation for the oblate case proposing the $\pi[310] 1 / 2 \otimes v[310] 1 / 2$ configuration for the ground state. The calculated magnetic moment for this configuration is 0.57 $\mu_{N}$ in good agreement with the previously measured experimental value.

The $\pi[301] 3 / 2 \otimes v[431] 3 / 2$ configuration is proposed for the $3^{-72 m} \mathrm{Br}$ state based on the configuration of the ${ }^{74 m} \mathrm{Br}$ that have a similar value for the magnetic moment. This configuration corresponds within the $\mathrm{HF}+\mathrm{BCS}$ (SLy4) framework to prolate deformed structure with a magnetic moment of 1.40 $\mu_{N}$ which is in agreement with the previously measured experimental value. This change of deformation from the ground state to the isomeric state in a small energy interval of only $101.2 \mathrm{keV}$ could explain the largely retarded $M 2$ transition. A possible $\beta$-decay branch from the $101.2-\mathrm{keV}$ state cannot be discarded.

Profiting from the electron spectrum we have determined that no $E 0$ transitions were observed in ${ }^{72} \mathrm{Br}$. However, we have measured the intensity of $E 0$ transitions in the isobaric partners ${ }^{72} \mathrm{Ge}$ and ${ }^{72} \mathrm{Se}$. For the ${ }^{72} \mathrm{Ge}$ case, we get a relative intensity of the $691-\mathrm{keV} E 0$ transition in the ${ }^{72} \mathrm{As} \rightarrow{ }^{72} \mathrm{Ge}$ decay that is a factor of 3 lower than previously measured and similar to the one obtained for ${ }^{72} \mathrm{Ga} \rightarrow{ }^{72} \mathrm{Ge}$ decay. For the ${ }^{72} \mathrm{Se}$ case, this is the first direct measurement of the $E 0$ transition intensity studied in the ${ }^{72} \mathrm{Br} \beta$ decay. We have also determined the relative branching of the $E 2$ and $E 0$ transitions deexciting the $937-\mathrm{keV}^{+}$state. The results on the intensity of the $E 0$ transition and the $E 0 / E 2$ ratio of intensities obtained in this work by conversion electron spectroscopy agree with previous ones obtained by $\gamma-\gamma$ coincidences.

\section{ACKNOWLEDGMENTS}

The authors of the IS370 collaboration want to acknowledge the support of the ISOLDE Collaboration and technical teams at CERN. J.A.B. acknowledges the predoctoral Grant No. BES-2008-009412 associated to the research Project No. FPA2007-62170 funded by Ministerio de Ciencia e Innovación (Spain). This work has been partly supported by the Spanish Funding Agency for Research (AEI) through Projects No. FPA2017-87568-P, No. RTI2018-098868-B-I00, No. PGC2018-093636-B-I00, No. PID2019-104390GB-I00, and No. PID2019-104714GB-C21 and by STFC (UK) through Grant No. ST/P005314/1.
[1] J. H. Hamilton et al., Phys. Rev. Lett. 32, 239 (1974).

[2] R. B. Piercey, J. H. Hamilton, R. Soundranayagam, A. V. Ramayya, C. F. Maguire, X. J. Sun, Z. Z. Zhao, R. L. Robinson, H. J. Kim, S. Frauendorf, J. Doring, L. Funke, G. Winter, J. Roth, L. Cleemann, J. Eberth, W. Neumann, J. C. Wells, J. Lin, A. C. Rester, and H. K. Carter, Phys. Rev. Lett. 47, 1514 (1981).

[3] S. Frauendorf and A. O. Macchiavelli, Prog. Part. Nucl. Phys. 78, 24 (2014).

[4] E. Ha, M. K. Cheoun, H. Sagawa, and W. Y. So, Phys. Rev. C 97, 064322 (2018).

[5] B. Cederwall et al., Phys. Rev. Lett. 124, 062501 (2020).

[6] A. Parikh et al., Prog. Part. Nucl. Phys. 69, 225 (2013).

[7] P. Sarriguren, Phys. Lett. B 680, 438 (2009).

[8] P. Sarriguren, Phys. Rev. C 83, 025801 (2011).

[9] H. Schmeing, J. C. Hardy, R. L. Graham, J. S. Geiger, and K. P. Jackson, Phys. Lett. B 44, 449 (1973).

[10] C. N. Davids and D. R. Goosman, Phys. Rev. C 8, 1029 (1973).

[11] I. Piqueras et al., Eur. Phys. J. A 16, 313 (2003).

[12] G. Garcia Bermudez, C. Baktash, A. J. Kreiner, and M. A. J. Mariscotti, Phys. Rev. C 25, 1396 (1982).

[13] S. Ulbig et al., Z. Phys. A At. Nucl. 329, 51 (1988).

[14] N. Fotiades, J. A. Cizewski, C. J. Lister, C. N. Davids, R. V. F. Janssens, D. Seweryniak, M. P. Carpenter, T. L. Khoo, T. Lauritsen, D. Nisius, P. Reiter, J. Uusitalo, I. Wiedenhover, A. O. Macchiavelli, and R. W. MacLeod, Phys. Rev. C 60, 057302 (1999).

[15] C. Plettner, I. Ragnarsson, H. Schnare, R. Schwengner, L. Kaubler, F. Donau, A. Algora, G. deAngelis, D. R. Napoli, A. Gadea, J. Eberth, T. Steinhardt, O. Thelen, M. Hausmann, A. Muller, A. Jungclaus, K. P. Lieb, D. G. Jenkins, R. Wadsworth, and A. N. Wilson, Phys. Rev. Lett. 85, 2454 (2000).
[16] C. D. O'Leary, R. Wadsworth, P. Fallon, C. E. Svensson, I. Ragnarsson, D. E. Appelbe, R. A. E. Austin, G. C. Ball, J. A. Cameron, M. P. Carpenter, R. M. Clark, M. Cromaz, M. A. Deleplanque, R. M. Diamond, D. F. Hodgson, R. V. F. Janssens, D. G. Jenkins, N. S. Kelsall, G. J. Lane, C. J. Lister, A. O. Macchiavelli, D. Sarantites, F. S. Stephens, D. Seweryniak, K. Vetter, J. C. Waddington, and D. Ward, Phys. Rev. C 69, 034316 (2004).

[17] R. Bengtsson, H. Frisk, F. R. May, and J. A. Pinston, Nucl. Phys. A 415, 189 (1984).

[18] R. Bengtsson and I. Ragnarsson, Nucl. Phys. A 436, 14 (1985).

[19] A. V. Afanasjev, D. B. Fossan, G. Lane, and I. Ragnarsson, Phys. Rep. 322, 1 (1999).

[20] A. G. Griffiths, C. J. Ashworth, J. Rikovska, N. J. Stone, J. P. White, I. S. Grant, P. M. Walker, and W. B. Walters, Phys. Rev. C 46, 2228 (1992).

[21] D. Abriola and A. A. Sonzogni, Nucl. Data Sheets 111, 1 (2010).

[22] W. E. Collins et al., Phys. Rev. C 9, 1457 (1974).

[23] E. Bouchez, I. Matea, W. Korten, F. Becker, B. Blank, C. Borcea, A. Buta, A. Emsallem, G. deFrance, J. Genevey, F. Hannachi, K. Hauschild, A. Hurstel, Y. LeCoz, M. Lewitowicz, R. Lucas, F. Negoita, F. de Oliveira Santos, D. Pantelica, J. Pinston, P. Rahkila, M. Rejmund, M. Stanoiu, and C. Theisen, Phys. Rev. Lett. 90, 082502 (2003).

[24] J. A. Briz, E. Nacher, M. J. G. Borge, A. Algora, B. Rubio, P. Dessagne, A. Maira, D. Cano-Ott, S. Courtin, D. Escrig, L. M. Fraile, W. Gelletly, A. Jungclaus, G. LeScornet, F. Marechal, C. Miehe, E. Poirier, A. Poves, P. Sarriguren, J. L. Tain, and O. Tengblad, Phys. Rev. C 92, 054326 (2015). 
[25] A. Gade, D. Bazin, A. Becerril, C. M. Campbell, J. M. Cook, D. J. Dean, D. C. Dinca, T. Glasmacher, G. W. Hitt, M. E. Howard, W. F. Mueller, H. Olliver, J. R. Terry, and K. Yoneda, Phys. Rev. Lett. 95, 022502 (2005).

[26] K. Wimmer et al., Eur. Phys. J. A 56, 159 (2020).

[27] J. van Klinken and K. Wisshak, Nucl. Instrum. Methods 98, 1 (1972); J. van Klinken, S. J. Feenstra, K. Wisshak, and H. Faust, ibid. 130, 427 (1975); J. van Klinken, S. J. Feenstra, and G. Dumont, ibid. 151, 433 (1978).

[28] A. B. Perez-Cerdan, B. Rubio, W. Gelletly, A. Algora, J. Agramunt, K. Burkard, W. Huller, E. Nacher, P. Sarriguren, L. Caballero, F. Molina, L. M. Fraile, E. Reillo, M. J. G. Borge, P. Dessagne, A. Jungclaus, and M. D. Salsac, Phys. Rev. C 84, 054311 (2011).

[29] J. Bea, Ph.D. thesis, Universidad de Valencia (1995).

[30] E. Roeckl et al., Z. Phys. 266, 123 (1974).

[31] A. Coban et al., Nucl. Phys. A 182, 385 (1972).

[32] M. J. Martin, Nucl. Data Sheets 70, 315 (1993).

[33] B. Singh et al., Nucl. Data Sheets 107, 1923 (2006).

[34] T. Kibédi et al., Nucl. Instrum. Methods A 589, 202 (2008)

[35] T. Paradellis et al., Nucl. Phys. A 201, 113 (1973).

[36] S. Agostinelli et al. Nucl. Instrum. Methods A 506, 250 (2003).

[37] W. J. Gallagher et al., Nucl. Instrum. Methods 122, 405 (1974).

[38] B. Jäckel et al., Nucl. Instrum. Methods A 261, 543 (1987).

[39] C. M. Mattoon, F. Sarazin, C. Andreoiu, A. N. Andreyev, R. A. E. Austin, G. C. Ball, R. S. Chakrawarthy, D. Cross, E. S. Cunningham, J. Daoud, P. E. Garrett, G. F. Grinyer, G. Hackman, D. Melconian, C. Morton, C. Pearson, J. J. Ressler, J. Schwarzenberg, M. B. Smith, and C. E. Svensson, Phys. Rev. C 80, 034318 (2009).

[40] J. A. Briz, PhD. thesis, Universidad Complutense de Madrid (2013).

[41] B. Singh et al., Nucl. Data Sheets 84, 487 (1998).

[42] C. G. Barham et al., Hyp. Int. 75, 431 (1992).
[43] A. A. Valverde, G. Bollen, K. Cooper, M. Eibach, K. Gulyuz, C. Izzo, D. J. Morrissey, R. Ringle, R. Sandler, S. Schwarz, C. S. Sumithrarachchi, and A. C. C. Villari, Phys. Rev. C 91, 037301 (2015).

[44] R. B. Firestone, V. S. Shirley, C. M. Baglin, S. Y. Frank Chu, and J. Zipkin, Table of Isotopes, 8th ed. (John Wiley \& Sons, New York, 1996).

[45] P. M. Endt, At. Data Nucl. Data Tables 23, 547 (1979).

[46] E. A. McCutchan, C. J. Lister, T. Ahn, R. J. Casperson, A. Heinz, G. Ilie, J. Qian, E. Williams, R. Winkler, and V. Werner, Phys. Rev. C 83, 024310 (2011).

[47] D. Kurath and R. D. Lawson, Phys. Rev. 161, 915 (1967).

[48] A. K. Jain, R. K. Sheline, D. M. Headly, P. C. Sood, D. G. Burke, I. Hrivnacova, J. Kvasil, D. Nosek, and R. W. Hoff, Rev. Mod. Phys. 70, 843 (1998).

[49] E. Chabanat, P. Bonche, P. Haensel, J. Meyer, and R. Schaeffer, Nucl. Phys. A 635, 231 (1998).

[50] P. Sarriguren, E. Moya de Guerra, and A. Escuderos, Nucl. Phys. A 658, 13 (1999); 691, 631 (2001).

[51] J. Kern and G. L. Struble, Nucl. Phys. A 286, 371 (1977).

[52] C. J. Gallagher and S. A. Moszkowski, Phys. Rev. 111, 1282 (1958).

[53] K. Abusaleem and B. Singh, Nucl. Data Sheets 112, 133 (2011).

[54] B. Singh and J. Chen, Nucl. Data Sheets 158, 1 (2019).

[55] A. G. Griffiths et al., Hyp. Int. 43, 481 (1988).

[56] M. Honma, T. Otsuka, T. Mizusaki, and M. Hjorth-Jensen, Phys. Rev. C 80, 064323 (2009).

[57] S. M. Lenzi, F. Nowacki, A. Poves, and K. Sieja, Phys. Rev. C 82, 054301 (2010).

[58] W. G. Wyckoff and J. E. Draper, Phys. Rev. C 8, 796 (1973).

[59] A. C. Rester, A. V. Ramayya, J. H. Hamilton, D. Krmpotic, and P. Venugopala Rao, Nucl. Phys. A 162, 461 (1971).

[60] A. C. Rester, J. H. Hamilton, A. V. Ramayya, and N. R. Johnson, Nucl. Phys. A 162, 481 (1971). 